\title{
Reversible Polymer-Chain Modification: Ring-Opening and Closing of Polylactone
}

\author{
Seunghwan Moon, Koichiro Masada, and Kyoko Nozaki* \\ Department of Chemistry and Biotechnology, Graduate School of Engineering, The \\ University of Tokyo, 7-3-1 Hongo, Bunkyo-ku, Tokyo 113-8656, Japan
}

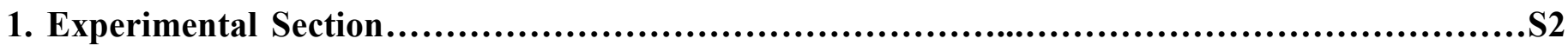

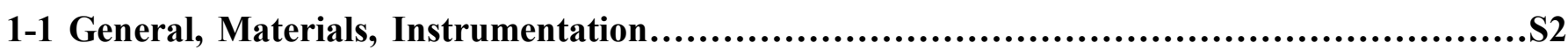

1-2 Details for the experimental procedures described in the main text..............................S2

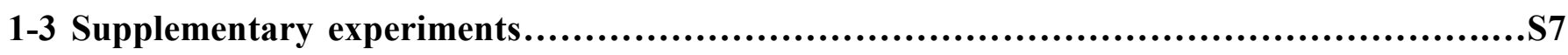

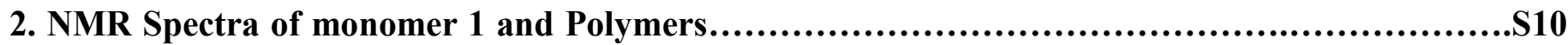

3. MALDI-TOF, SEC Charts and DSC Charts of Polymers..............................................S18 


\section{Experimental Procedures}

\section{1-1 General, Materials, Instrumentation}

\section{General}

All reactions and polymerizations were carried out using a standard glovebox or Schlenk techniques under argon purified by passing through a hot column packed with BASF catalyst R3-11.

\section{Material}

Carbon dioxide ( $>99.990$ vol\%) was purchased from Takachiho Chemical Industrial Co. (Takachiho) and used after passing through a dry column DC-HDF300-A3 made by Nikka Seiko Co., Ltd. 1,3-Butadiene (>99\%) was purchased from Takachiho and used as received. Dehydrated acetonitrile, and tetrahydrofuran were purchased from Kanto Chemical Co. Inc. (Kanto) and used as received. Benzylamine (TCI, >99\%), and triethylamine (TCI, >99\%) were distilled before use. Tetrabutylammonium acetate (TBAAc) (>97\%) was purchased from Combi-blocks and used as received. The other reagents were purchased from TCI, Kanto, Sigma-Aldrich Chemical Co. or Wako Pure Chemical Industries, Ltd. and were used as received.

\section{Instrumentation}

NMR spectra were recorded on 400 and $500 \mathrm{MHz}$ spectrometers (JEOL JNM-ECS400 or BRUKER Ascend500) at ambient temperature unless otherwise noted. Chemical shift values for protons are referenced to the residual proton resonance of DMSO- $d_{6}\left(\mathrm{CD}_{3} \mathrm{SOCD}_{3}, \delta: 2.50\right)$, chloroform- $d\left(\mathrm{CDCl}_{3}, \delta: 7.26\right)$ or o-dichlorobenzene- $d_{4}\left(\mathrm{C}_{6} \mathrm{D}_{4} \mathrm{Cl}_{2}, \delta: 6.94\right)$. Infrared (IR) spectra were recorded on a Shimadzu FTIR-8400 equipped with an attenuated total reflection (ATR) system. Mass spectra (MS) were measured with a matrix-assisted laser desorption/ionization (MALDI) method on a Bruker autoflex speed mass spectrometer. Size-exclusion chromatography (SEC) analyses were carried with two columns (Shodex KF-804L) using 10 $\mathrm{mM} \mathrm{LiBr}$ solution of dimethylformamide as an eluent at $40{ }^{\circ} \mathrm{C}$ at $1 \mathrm{~mL} / \mathrm{min}$. The molecular weight was calibrated against standard polystyrene samples. Differential scanning calorimetry (DSC) measurements of polymers were performed on a Seiko DSC 7020 analyzer at a heating and cooling rate of $20{ }^{\circ} \mathrm{C} / \mathrm{min}$. Glass-transition temperature ( $\mathrm{Tg}$ ) values were determined from the third heating scan. Thermogravimetric (TG) analyses were performed on a Seiko EXSTAR 6000 TG/DTA 6200 analyzer at a heating rate of $10^{\circ} \mathrm{C} / \mathrm{min}$.

\section{1-2 Details for the experimental procedures described in the main text Preparation of 3-ethylidene-6-vinyltetrahydro-2H-Pyran-2-one 1}

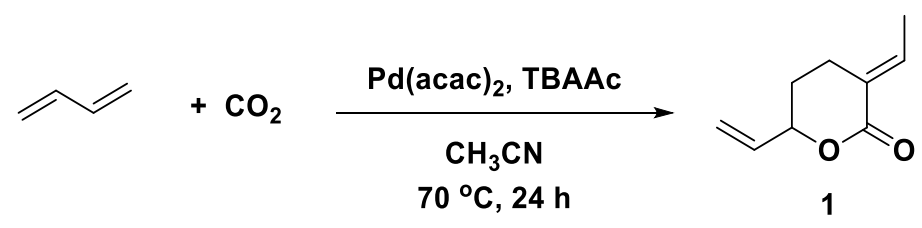

Synthesized by using reported procedure. To a $50-\mathrm{mL}$ stainless autoclave with a magnetic stirring bar, $\operatorname{Pd}(\mathrm{acac})_{2}(45.6 \mathrm{mg}$, $0.15 \mathrm{mmol})$, and TBAAc $(0.906 \mathrm{~g}, 3.0 \mathrm{mmol})$ were charged with $\mathrm{CH}_{3} \mathrm{CN}(10 \mathrm{~mL})$. To the mixture was added 1,3-butadiene $(17 \mathrm{~g}, 0.32 \mathrm{~mol})$ at $-20{ }^{\circ} \mathrm{C}$ over 20 minutes with stirring. Then the autoclave was pressurized with $4.0 \mathrm{MPa}$ of $\mathrm{CO}_{2}$ for 3 minutes. After stirred at $70{ }^{\circ} \mathrm{C}$ for $24 \mathrm{~h}$, the autoclave was cooled to room temperature with a water bath. After residual gas pressure was released, the volatile matters were removed by evaporation. The obtained oil was purified by column chromatography on silica gel (hexane/ethyl acetate $=3 / 1$ ). After the solvents were removed, the product was purified by 
distillation; yield: $7.8 \mathrm{~g}(33 \%)$

${ }^{1} \mathrm{H}$ NMR $\left(400 \mathrm{MHz}, \mathrm{CDCl}_{3}\right) \delta 1.56-1.68(\mathrm{~m}, 4 \mathrm{H}), \delta 1.88-1.98(\mathrm{~m}, 1 \mathrm{H}), \delta 2.26-2.36(\mathrm{~m}, 1 \mathrm{H}), \delta 2.42-2.51(\mathrm{~m}, 1 \mathrm{H}), \delta 4.61-$

$4.68(\mathrm{~m}, 1 \mathrm{H}), \delta$ 5.06-5.12 (m, 1H), 5.15-5.26 (m, 1H), 5.69-5.80 (m, 1H), 6.92-7.02 (m, 1H)

\section{Polymerization of lactone 1}

A mixture of lactone 1 (7.00 g, $46.0 \mathrm{mmol}$ ) and V-40 (1,1'-azobis(cyclohexanecarbonitrile)) (112 mg 0.458 mmol) was stirred at $100{ }^{\circ} \mathrm{C}$ for three days in a $25 \mathrm{~mL}$ Schlenk tube. The resulting mixture was diluted with an excess amount of methanol, and the formed precipitate was collected and washed with methanol. After drying under vacuum, the crude materials were dissolved in hot DMF and reprecipitated with aqueous hydrochloric acid (1.0 M) to afford 2 (2.14 g).

\section{Hydrolysis of polymer 2}

To the mixture of $2(50 \mathrm{mg})$, and THF $(1.0 \mathrm{~mL})$, aqueous solution of $\mathrm{KOH}(0.20 \mathrm{~g}$ in $4.6 \mathrm{~mL})$ was added and the mixture was stirred at $100{ }^{\circ} \mathrm{C}$ for 3 days in a $50 \mathrm{~mL}$ autoclave. The resulting mixture was diluted with excess amount of aqueous hydrochloric acid (1.0 M), and the formed suspension was centrifugated (4000 rpm, $5 \mathrm{~min}$.). The supernatant liquid was decanted, and the precipitate was washed with excess amount of water. The precipitate was isolated by centrifugation or filtration. The obtained product 3a was dried under vacuum at $100{ }^{\circ} \mathrm{C}$ for 5 hours.

\section{Aminolysis of polymer 2 with TBD}

The mixture of $2(200 \mathrm{mg})$, benzylamine $(5.4 \mathrm{~mL}, 49 \mathrm{mmol})$, and 1,5,7-triazabicyclo[4.4.0]dec-5-ene (TBD, $92 \mathrm{mg}, 0.66$ $\mathrm{mmol}$ ) was stirred in a $5 \mathrm{~mL} \mathrm{Schlenk}$ tube at $100{ }^{\circ} \mathrm{C}$, for 3 days. The resulting mixture was diluted with an excess amount of $\mathrm{Et}_{2} \mathrm{O}$, and the formed precipitate was collected and washed with $\mathrm{Et}_{2} \mathrm{O}$. After drying under vacuum, the crude materials were dissolved in DMF and reprecipitated with aqueous hydrochloric acid (1.0 M) to afford $\mathbf{3} \mathbf{b}_{\mathbf{1}}$. The obtained product was dried under vacuum at $100{ }^{\circ} \mathrm{C}$ for 5 hours.

\section{Aminolysis of polymer 2 with BnNHLi}

To the mixture of benzylamine $(0.10 \mathrm{~mL}, 0.92 \mathrm{mmol})$ and THF $(0.1 \mathrm{~mL}), 2.6 \mathrm{M}$ solution of $n$-butyllithium in hexane $(0.35$ $\mathrm{mL}, 0.91 \mathrm{mmol}$ ) was added at $-78{ }^{\circ} \mathrm{C}$ and the mixture was stirred at the same temperature for minutes in a $5 \mathrm{~mL}$ Schlenk tube. The solution of $2(50 \mathrm{mg})$ in THF $(0.5 \mathrm{~mL})$ was added to the mixture and the mixture was warmed to room temperature slowly. The mixture was stirred for $24 \mathrm{~h}$ at room temperature, and the reaction mixture was quenched with small amount of $\mathrm{MeOH}$ and diluted with excess amount of $\mathrm{Et}_{2} \mathrm{O}$. The formed precipitate was filtered and dried under vacuum. The crude product was dissolved into hot DMF, and reprecipitated with aqueous hydrochloric acid (1.0 M) to afford $\mathbf{3} \mathbf{b}_{\mathbf{2}}$. The obtained product was dried under vacuum at $100{ }^{\circ} \mathrm{C}$ for 5 hours.

\section{Ring-closing reaction of polymer 3a (by heating)}

The Schlenk tube with $10 \mathrm{mg}$ of $\mathbf{3 a}$ was heated at $180{ }^{\circ} \mathrm{C}$ for 45 minutes under the flow of $\mathrm{N}_{2}$.

\section{Ring-closing reaction of polymer 3a (by organic reagent)}

To the mixture of 3a $(50 \mathrm{mg})$, THF $(0.5 \mathrm{~mL})$, and HMPA $(0.5 \mathrm{~mL})$, triethylamine $(0.24 \mathrm{~mL}, 1.7 \mathrm{mmol})$ was added and the mixture was stirred at room temperature in a $5 \mathrm{~mL}$ Schlenk tube. After 30 minutes, tosyl chloride $(170 \mathrm{mg}, 0.88 \mathrm{mmol})$ was added and the mixture was stirred at $60{ }^{\circ} \mathrm{C}$ for $24 \mathrm{~h}$. The mixture was dissolved into small amount of DMF, and by pouring 
excess amount of aqueous hydrochloric acid $(1.0 \mathrm{M})$, precipitate was formed and isolated by filtration. After drying under vacuum, the crude materials were dissolved in small amount of THF and reprecipitated with excess amount of $\mathrm{Et}_{2} \mathrm{O}$. The obtained product was dried under vacuum at $100{ }^{\circ} \mathrm{C}$ for 5 hours.

\section{Examined ring-closing reaction of polymers $3 b_{1}$ and $3 b_{2}$}
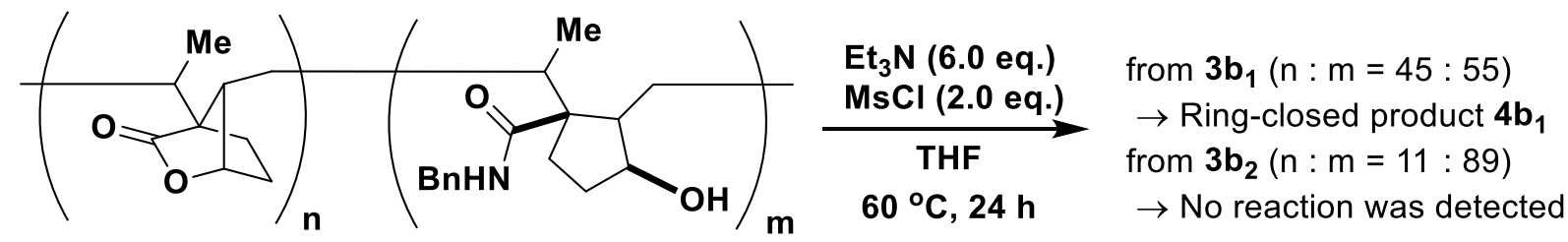

To the mixture of $\mathbf{3} \mathbf{b}_{1}$ or $\mathbf{3} \mathbf{b}_{2}(25 \mathrm{mg})$ and THF $(0.5 \mathrm{~mL})$, triethylamine $(0.060 \mathrm{~mL}, 0.43 \mathrm{mmol})$ was added and the mixture was stirred at room temperature in a $5 \mathrm{~mL}$ Schlenk tube. After 30 minutes, mesyl chloride $(0.010 \mathrm{~mL}, 0.13 \mathrm{mmol})$ was added and the mixture was stirred at $60{ }^{\circ} \mathrm{C}$ for $24 \mathrm{~h}$. The mixture was dissolved into small amount of THF, and by pouring excess amount of $\mathrm{Et}_{2} \mathrm{O}$, precipitate was formed and isolated by filtration. After drying under vacuum, the crude materials were dissolved in small amount of THF and reprecipitated with excess amount of $\mathrm{Et}_{2} \mathrm{O}$. The obtained product was dried under vacuum at $100{ }^{\circ} \mathrm{C}$ for 5 hours.

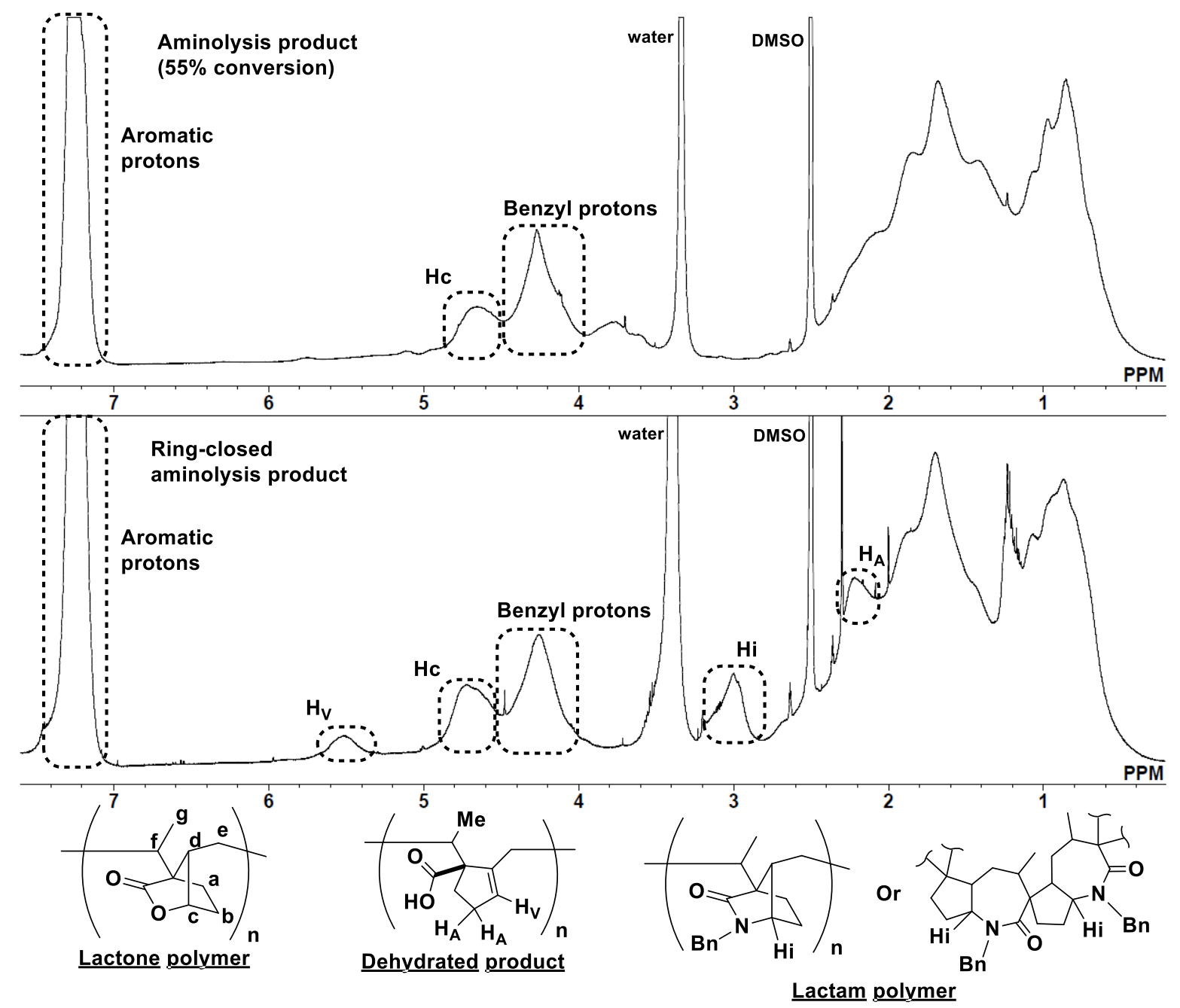

Figure S1. ${ }^{1} \mathrm{H}$ NMR spectra of the aminolysis product $\mathbf{3 b}_{\mathbf{1}}$ (with $55 \%$ conversion) and ring-closed product $\mathbf{4} \mathbf{b}_{\mathbf{1}}(500 \mathrm{MHz}$, DMSO- $\left.d_{6}\right)$. 


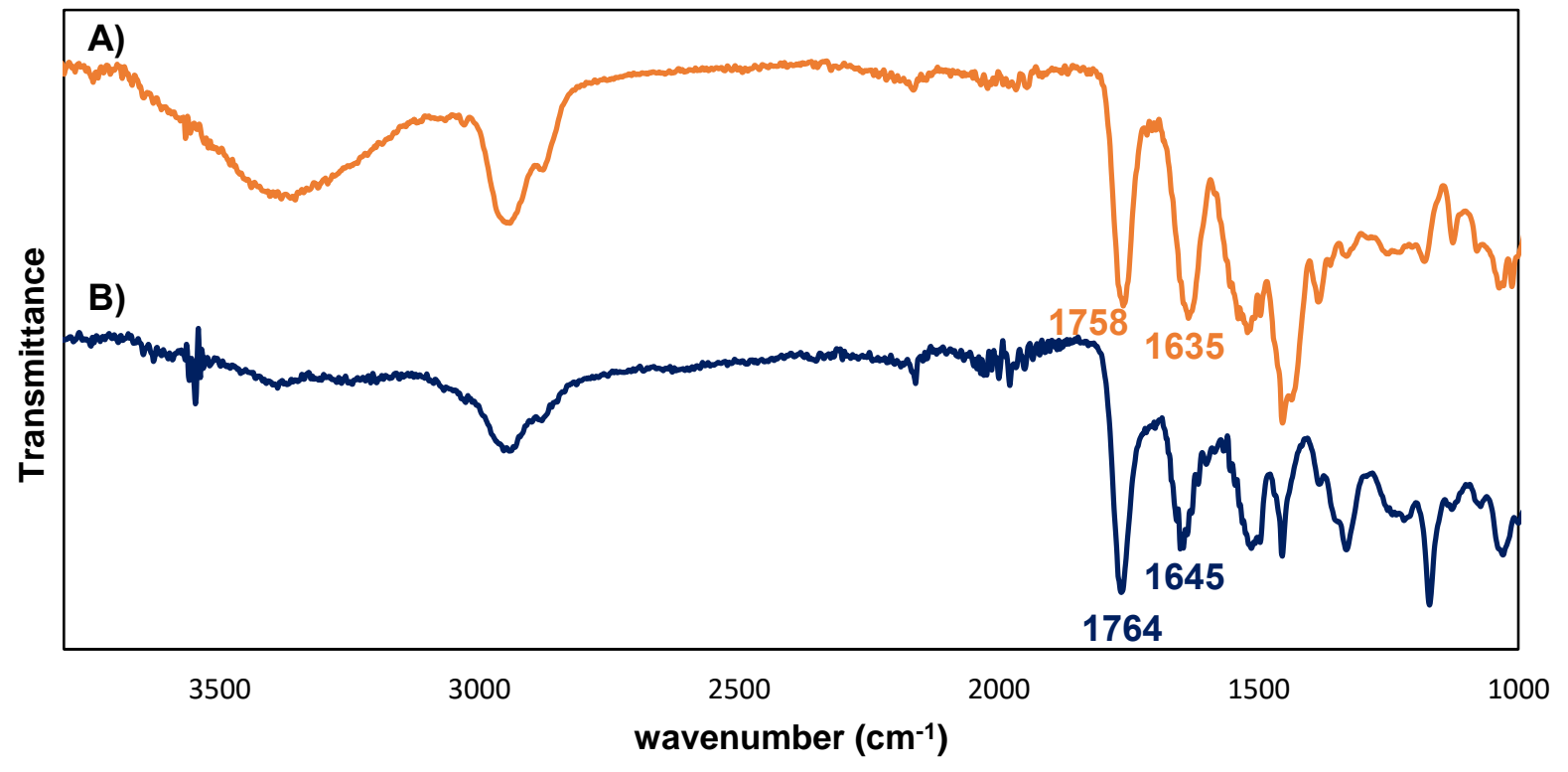

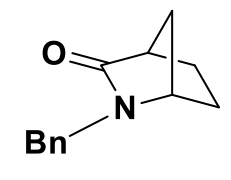

$\mathrm{C}=\mathrm{O}$ stretching $\mathrm{C}=\mathrm{O}$ stretching : $1693 \mathrm{~cm}^{-1}$

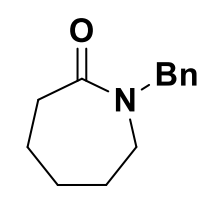

: $1637 \mathrm{~cm}^{-1}$<smiles>O=C1CC2CCCCC2CCN1CCO</smiles>

$\mathrm{C}=\mathbf{0}$ stretching : $1643 \mathrm{~cm}^{-1}$<smiles>[X]CCC1CCCC(=O)N1CCO</smiles>

$\mathrm{C}=\mathrm{O}$ stretching : $1644 \mathrm{~cm}^{-1}$

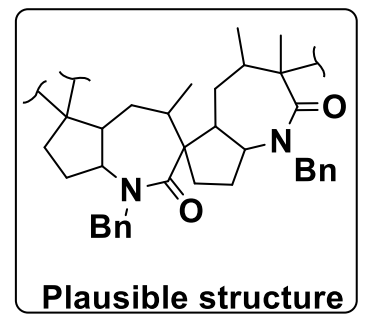

Figure S2. IR spectra of the aminolysis product $\mathbf{3} \mathbf{b}_{\mathbf{1}}$ (with $55 \%$ conversion) (A), and ring-closed product $\mathbf{5} \mathbf{b}_{\mathbf{1}}(\mathrm{B})$, and $\mathrm{C}=\mathrm{O}$ stretching of the related compounds.

\section{Possible explanation of the results of ring-closing reaction of $3 b_{1}$ and $3 b_{2}$}

From the attempted experiments to close the ring of $\mathbf{3} \mathbf{b}_{\mathbf{1}}$ and $\mathbf{3} \mathbf{b}_{\mathbf{2}}$, which have different ratio of the bicyclic lactone moieties and ring-opened amide moieties, the ring-closed product was obtained only from $\mathbf{3} \mathbf{b}_{\mathbf{1}}$. To find out the reason of these results, we tried ${ }^{1} \mathrm{H}$ NMR measurement of each aminolysis product using o-dichlorobenzene- $d_{4}$ as the solvent and checked the hydrogen bond of the polymers. It was found out that in case of $\mathbf{3 b}_{\mathbf{1}}, \mathrm{N}-\underline{\mathrm{H}}$ and $\mathrm{O}-\underline{\mathrm{H}}$ are observed as one peak at $2.38 \mathrm{ppm}$ judging from the integration of the disappeared peak after $\mathrm{D}_{2} \mathrm{O}$ addition, and it is possibly due to the fast exchange of these protons via hydrogen bond (Figure S3).

The same measurement of the ${ }^{1} \mathrm{H}$ NMR of $3 \mathbf{b}_{2}$ before and after the addition of $\mathrm{D}_{2} \mathrm{O}$ gave different results. No changes of the peaks were observed even after $\mathrm{D}_{2} \mathrm{O}$ addition, possibly indicating stronger hydrogen bond, which eventually made $\mathrm{H} / \mathrm{D}$ exchange much more difficult (Figure S4). From these results, we reasoned that the ring-closing reaction proceeded only with $\mathbf{3 b}_{\mathbf{1}}$ because of the less tight hydrogen bond, leading to room for reaction with methanesulfonyl chloride, and further, the ringclosing reaction. The same reaction with $\mathbf{3} \mathbf{b}_{2}$ gave neither ring-closed moieties nor dehydrated moieties, indicating that even the mesylation of the hydroxy groups did not occur. 


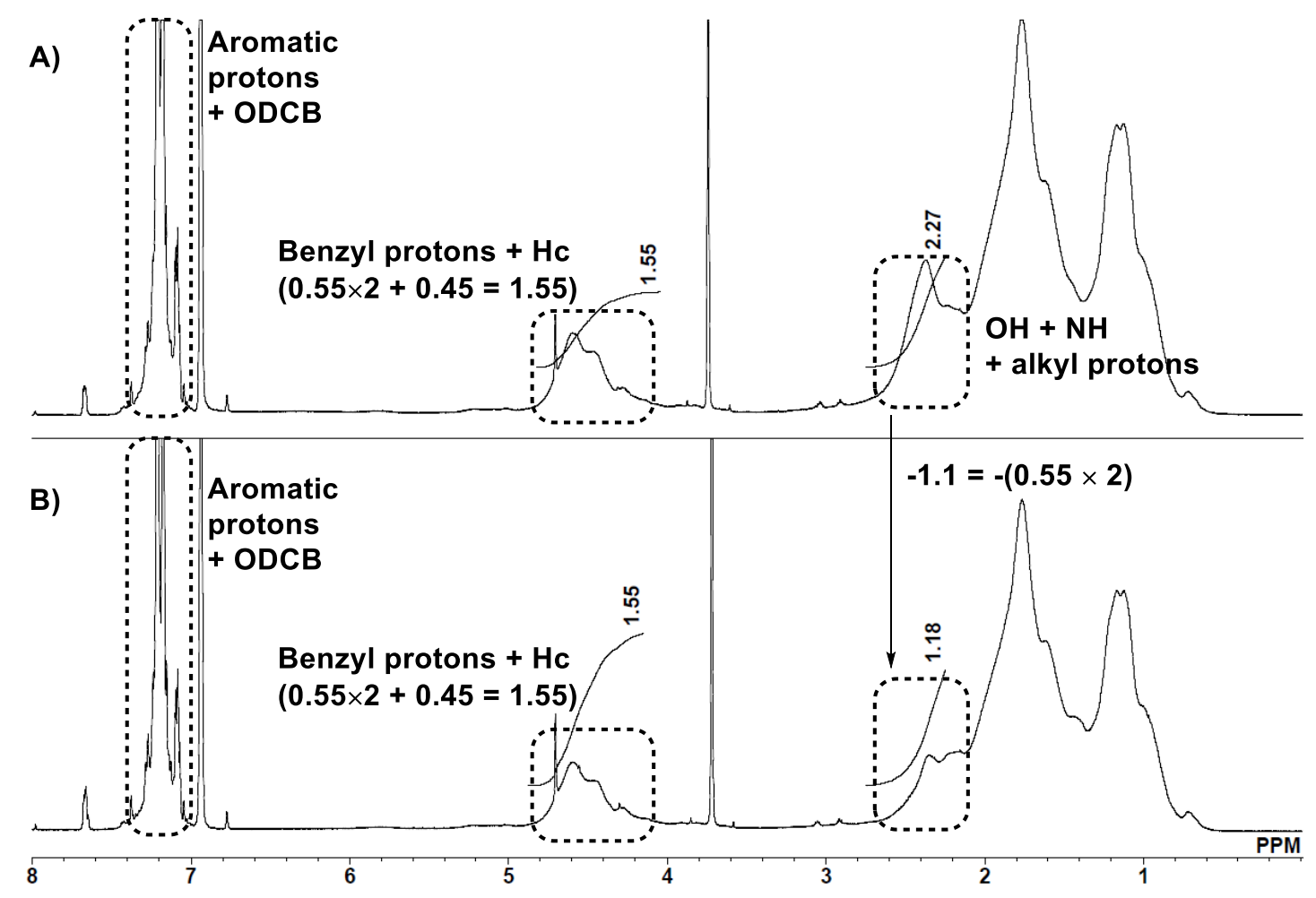

Figure S3. ${ }^{1} \mathrm{H}$ NMR spectra of the aminolysis product $\mathbf{3} \mathbf{b}_{1}$ (with $55 \%$ conversion) before (A) and after (B) $\mathrm{D}_{2} \mathrm{O}$ addition $\left(500 \mathrm{MHz}, 120{ }^{\circ} \mathrm{C}\right.$, o-dichlorobenzene(ODCB)- $\left.d_{4}\right)$

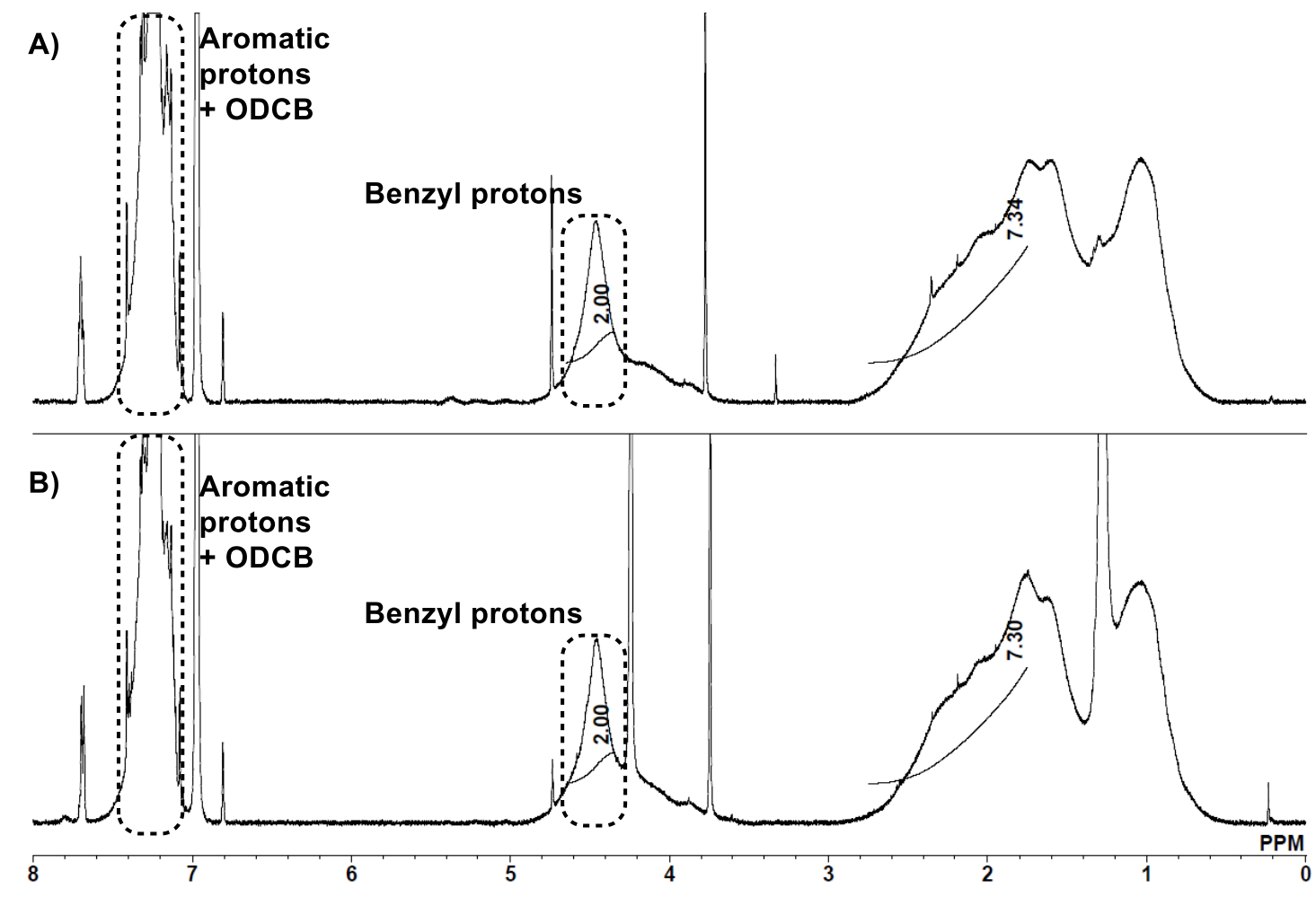

Figure S4. ${ }^{1} \mathrm{H}$ NMR spectra of the aminolysis product $\mathbf{3} \mathbf{b}_{\mathbf{2}}$ (with $89 \%$ conversion) before (A) and after (B) $\mathrm{D}_{2} \mathrm{O}$ addition (500 MHz, $120{ }^{\circ} \mathrm{C}$, o-dichlorobenzene(ODCB)- $d_{4}$ ) 


\section{1-3 Supplementary experiments}

Screening of the hydrolysis of 2

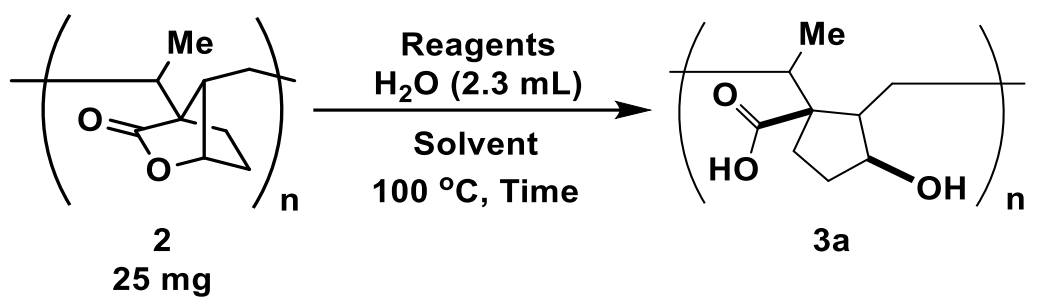

Table S1

\begin{tabular}{|c|c|c|c|c|c|}
\hline Entry & Reagents & Solvent $(\mathrm{mL})$ & Temperature & Time & Conversion* \\
\hline 1 & $\mathrm{CF}_{3} \mathrm{COOH} 10$ eq. & $\operatorname{DCM}(0.8)$ & $\mathrm{rt}$ & $20 \mathrm{~h}$ & NR \\
\hline 2 & $\mathrm{HCl} 10$ eq. $(1 \mathrm{M})$ & DCE $(0.3)$ & $80^{\circ} \mathrm{C}$ & $24 \mathrm{~h}$ & NR \\
\hline 3 & $\mathrm{KOH} 10$ eq. & DMF (2.8) & $90^{\circ} \mathrm{C}$ & $24 \mathrm{~h}$ & $9 \%$ \\
\hline 4 & $\mathrm{KOH} 10$ eq. & 1,4-dioxane $(0.5)$ & $90^{\circ} \mathrm{C}$ & $42 \mathrm{~h}$ & $27 \%$ \\
\hline 5 & $\mathrm{NaOH} 10$ eq. & 1,4-dioxane $(0.5)$ & $100^{\circ} \mathrm{C}$ & $24 \mathrm{~h}$ & $12 \%$ \\
\hline 6 & KOH 10 eq., 18 -crown-6 10 eq. & THF (1.0) & $65^{\circ} \mathrm{C}$ & $24 \mathrm{~h}$ & $50 \%$ \\
\hline $7 * *$ & $\mathrm{KOH} 10$ eq., 18 -crown-6 1.0 eq. & THF (1.0) & $100{ }^{\circ} \mathrm{C}$ & $72 \mathrm{~h}$ & $90 \%$ \\
\hline
\end{tabular}

${ }^{*}$ Conversion was calculated based on ${ }^{1} \mathrm{H}$ NMR. ** Reaction using an autoclave *** DCE $=1,2$-dichloroethane

Screening of the aminolysis of 2

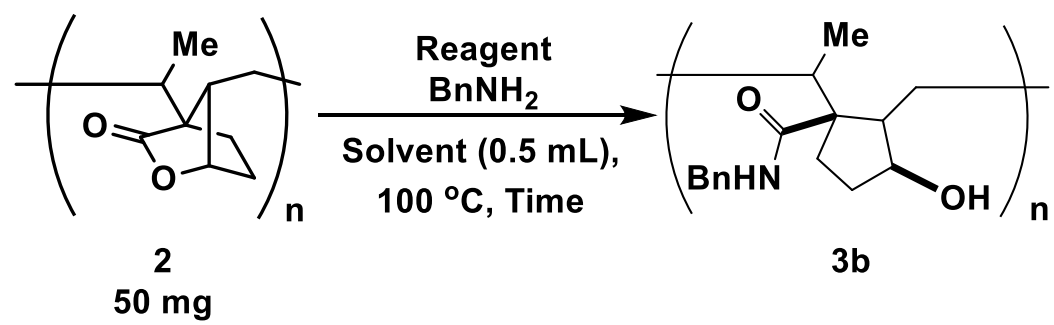

Table S2

\begin{tabular}{ccccccc}
\hline Entry & Reagent & $\mathrm{BnNH}_{2}(\mathrm{~mL})$ & Solvent & Temperature & Time & Conversion* \\
\hline 1 & $\mathrm{Sc}(\mathrm{OTf})_{3} 5 \mathrm{~mol} \%$ & 1.8 & Neat & $100{ }^{\circ} \mathrm{C}$ & $24 \mathrm{~h}$ & $23 \%$ \\
2 & $\mathrm{Y}(\mathrm{OTf})_{3} 5 \mathrm{~mol} \%$ & 1.8 & Neat & $100^{\circ} \mathrm{C}$ & $24 \mathrm{~h}$ & $20 \%$ \\
3 & $\mathrm{TBD} \mathrm{30} \mathrm{mol} \%$ & 1.8 & Neat & $100^{\circ} \mathrm{C}$ & $24 \mathrm{~h}$ & $21 \%$ \\
$4^{\#}$ & $\mathrm{TBD} \mathrm{30} \mathrm{mol} \%$ & 25 & Neat & $100^{\circ} \mathrm{C}$ & $72 \mathrm{~h}$ & $55 \%$ \\
5 & $n$-BuLi 1.0 eq. & 0.10 & THF & $25{ }^{\circ} \mathrm{C}$ & $24 \mathrm{~h}$ & $50 \%$ \\
6 & $n$-BuLi 2.8 eq. & 0.10 & THF & $25{ }^{\circ} \mathrm{C}$ & $24 \mathrm{~h}$ & $89 \%$ \\
\hline
\end{tabular}

* Conversion was calculated based on ${ }^{1} \mathrm{H}$ NMR. $* * \mathrm{TBD}=1,5,7$-triazabicyclo[4.4.0]dec-5-ene $\quad \# 1.0 \mathrm{~g}$ of 2 was used. 


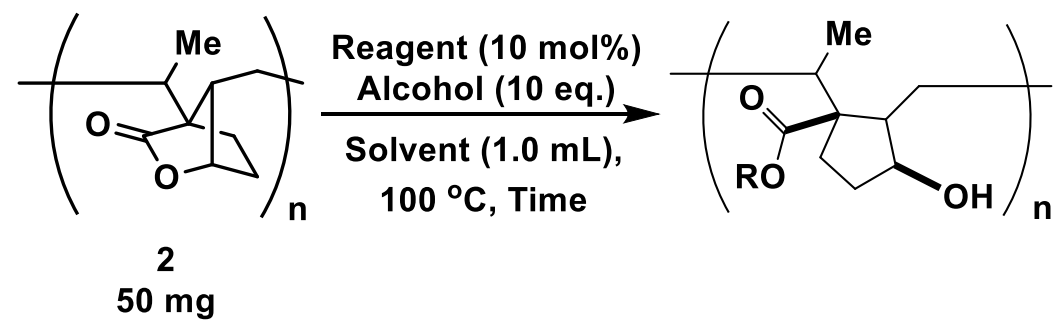

Table S3

\begin{tabular}{ccccccc}
\hline Entry & Alcohol & Reagent & Solvent & Temperature & Time & Conversion* \\
\hline 1 & Furfuryl alcohol & TBD $30 \mathrm{~mol} \%$ & THF & $65^{\circ} \mathrm{C}$ & $24 \mathrm{~h}$ & $\mathrm{NR}$ \\
2 & Furfuryl alcohol & TBD 30 mol\% & Neat & $100^{\circ} \mathrm{C}$ & $24 \mathrm{~h}$ & $\mathrm{NR}$ \\
3 & Furfuryl alcohol & $\mathrm{Ti}(\mathrm{O} i \mathrm{Pr})_{4}$ & Neat & $100^{\circ} \mathrm{C}$ & $24 \mathrm{~h}$ & $\mathrm{NR}$ \\
4 & Furfuryl alcohol & $\mathrm{Al}(\mathrm{O} i \mathrm{Pr})_{3}$ & Neat & $100^{\circ} \mathrm{C}$ & $72 \mathrm{~h}$ & $\mathrm{NR}$ \\
5 & 4-methoxybenzyl alcohol & $\mathrm{Ti}(\mathrm{O} i \mathrm{Pr})_{4}$ & Neat & $100^{\circ} \mathrm{C}$ & $24 \mathrm{~h}$ & $\mathrm{NR}$ \\
6 & 4-methoxybenzyl alcohol & $\mathrm{Al}(\mathrm{O} i \mathrm{Pr})_{3}$ & Neat & $100^{\circ} \mathrm{C}$ & $24 \mathrm{~h}$ & $\mathrm{NR}$ \\
\hline
\end{tabular}

* Conversion was calculated based on ${ }^{1} \mathrm{H}$ NMR. NR means No reaction.

Ring-closing of 3a by sulfonyl chlorides

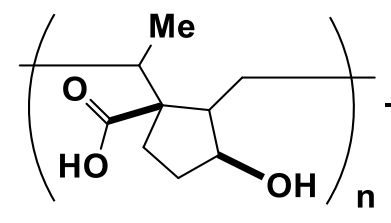

3a

$25 \mathrm{mg}$

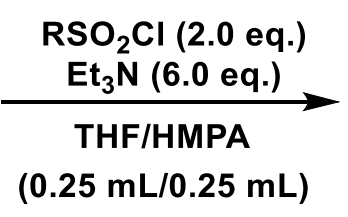

$60^{\circ} \mathrm{C}, 24 \mathrm{~h}$

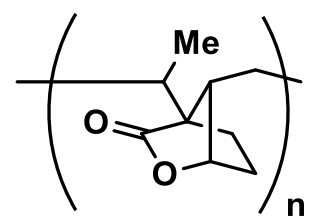

2'

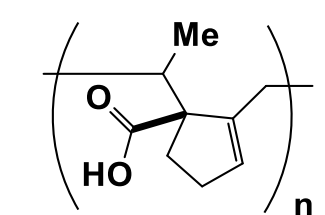

Dehydrated product

Table S4

\begin{tabular}{ccccc}
\hline \multirow{2}{*}{ Entry } & \multirow{2}{*}{$\mathrm{RSO}_{2} \mathrm{Cl}$} & Conversion (\%) & \multicolumn{2}{c}{ Results (\%) } \\
\cline { 4 - 5 } & & & $\mathbf{2}$ & Dehydrated product \\
\hline 1 & $\mathrm{MsCl}$ & 100 & 80 & 20 \\
2 & $\mathrm{TsCl}$ & 100 & 100 & 0 \\
\hline
\end{tabular}


Attempted reactions for lactam formation starting from $3 b_{1}$

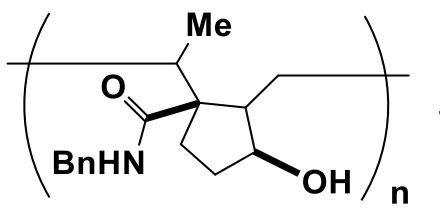

$3 b_{1}$

$50 \mathrm{mg}$

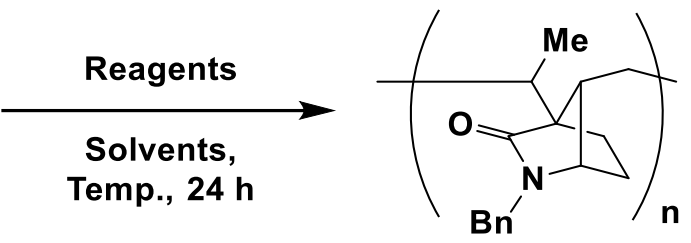

$\mathrm{Bn}^{\prime}$

Table S5

\begin{tabular}{|c|c|c|c|c|}
\hline \multirow{2}{*}{ Entry } & Reagents & \multirow{2}{*}{$\begin{array}{l}\text { Temp. } \\
\left({ }^{\circ} \mathrm{C}\right)\end{array}$} & \multirow{2}{*}{$\begin{array}{l}\text { Yield } \\
(\mathrm{mg})\end{array}$} & \multirow{2}{*}{ Results } \\
\hline & Solvents & & & \\
\hline \multirow{2}{*}{$1 *$} & $\mathrm{Tf}_{2} \mathrm{O}$ (2.2 eq.) & \multirow{2}{*}{60} & \multirow{2}{*}{40} & \multirow{2}{*}{$\begin{array}{c}\text { Almost no conversion, small amount of } \\
\text { dehydrated product }\end{array}$} \\
\hline & $\operatorname{DCE}(1.0 \mathrm{~mL})$ & & & \\
\hline \multirow{2}{*}{2} & Trifluoroacetic anhydride (2.2 eq.) & \multirow{2}{*}{$\mathrm{rt}$} & \multirow{2}{*}{50} & \multirow{2}{*}{ No conversion } \\
\hline & DCM $(1.0 \mathrm{~mL})$ & & & \\
\hline \multirow{2}{*}{3} & $\mathrm{HCl}(4.5 \mathrm{M}, 1.0 \mathrm{~mL})$ & \multirow{2}{*}{80} & \multirow{2}{*}{34} & \multirow{2}{*}{ Converted to 2} \\
\hline & 1,4-dioxane $(0.3 \mathrm{~mL})$ & & & \\
\hline \multirow{2}{*}{4} & TsOH (20 mol \%) & \multirow{2}{*}{80} & \multirow{2}{*}{49} & \multirow{2}{*}{ No conversion } \\
\hline & Chlorobenzene $(1.0 \mathrm{~mL})$ & & & \\
\hline \multirow{3}{*}{5} & ${ }^{t} \mathrm{BuOK}$ (2.2 eq.), $\mathrm{TsCl}$ (1.2 eq.), & \multirow{3}{*}{60} & \multirow{3}{*}{ n.a } & \multirow{3}{*}{ Small amount of dehydrated product } \\
\hline & LiI (1.2 eq.) & & & \\
\hline & HMPA (0.2 mL), THF (1.0 mL) & & & \\
\hline \multirow{3}{*}{6} & $\mathrm{KOH}$ (2.2 eq.), $\mathrm{TsCl}$ (1.2 eq.), & \multirow{3}{*}{60} & \multirow{3}{*}{36} & \multirow{3}{*}{ No conversion } \\
\hline & LiI (1.2 eq.) & & & \\
\hline & HMPA (0.2 mL), THF (1.0 mL) & & & \\
\hline \multirow{3}{*}{7} & $\mathrm{KOH}$ (2.2 eq.), $\mathrm{MsCl}$ (1.2 eq.), & \multirow{3}{*}{60} & \multirow{3}{*}{44} & \multirow{3}{*}{ No conversion } \\
\hline & LiI (1.2 eq.) & & & \\
\hline & THF (1.0 mL) & & & \\
\hline \multirow{3}{*}{8} & $\mathrm{KOH}$ (2.2 eq.), $\mathrm{MsCl}$ (1.2 eq.), & & & \\
\hline & LiI (1.2 eq.) & 60 & 47 & Small amount of dehydrated product \\
\hline & DMSO $1.0 \mathrm{~mL}$ & & & \\
\hline & $\mathrm{SOCl}_{2}$ (1.1 eq.), Pyridine $(0.05 \mathrm{~mL})$ & & 15 & No nonumerion \\
\hline F & $\mathrm{DCM}(0.6 \mathrm{~mL})$ & 11 & 更 & 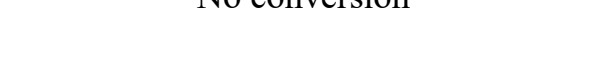 \\
\hline 10 & $\mathrm{CBr}_{4}$ (2.0 eq.), $\mathrm{PPh}_{3}$ (2.0 eq.) & to 60 & 15 & Smoll omount of dobudroted nroduct \\
\hline 10 & THF (1.0 mL) & 10000 & (1) & 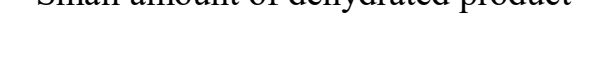 \\
\hline & $\mathrm{CBr}_{4}$ (2.0 eq.), $\mathrm{PPh}_{3}$ (2.0 eq.) & & & \\
\hline 11 & DMSO (1.0 mL) & 00 & 33 & 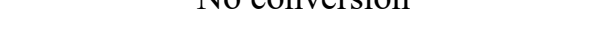 \\
\hline
\end{tabular}

* Results of the reactions were confirmed by ${ }^{1} \mathrm{H}$ NMR. ** $\mathrm{DCE}=1$,2-dichloroethane 
d, $\mathbf{g}$<smiles>C=CC1CCC(=CC)C(=O)O1</smiles>

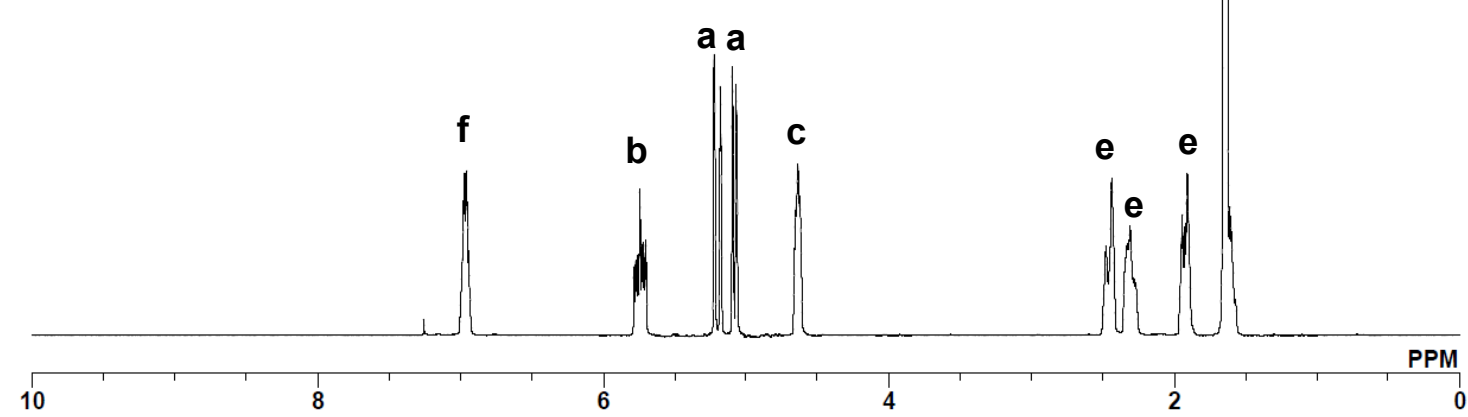

Figure S5. ${ }^{1} \mathrm{H}$ NMR spectrum $\left(400 \mathrm{MHz}, \mathrm{CDCl}_{3}\right)$ of lactone 1

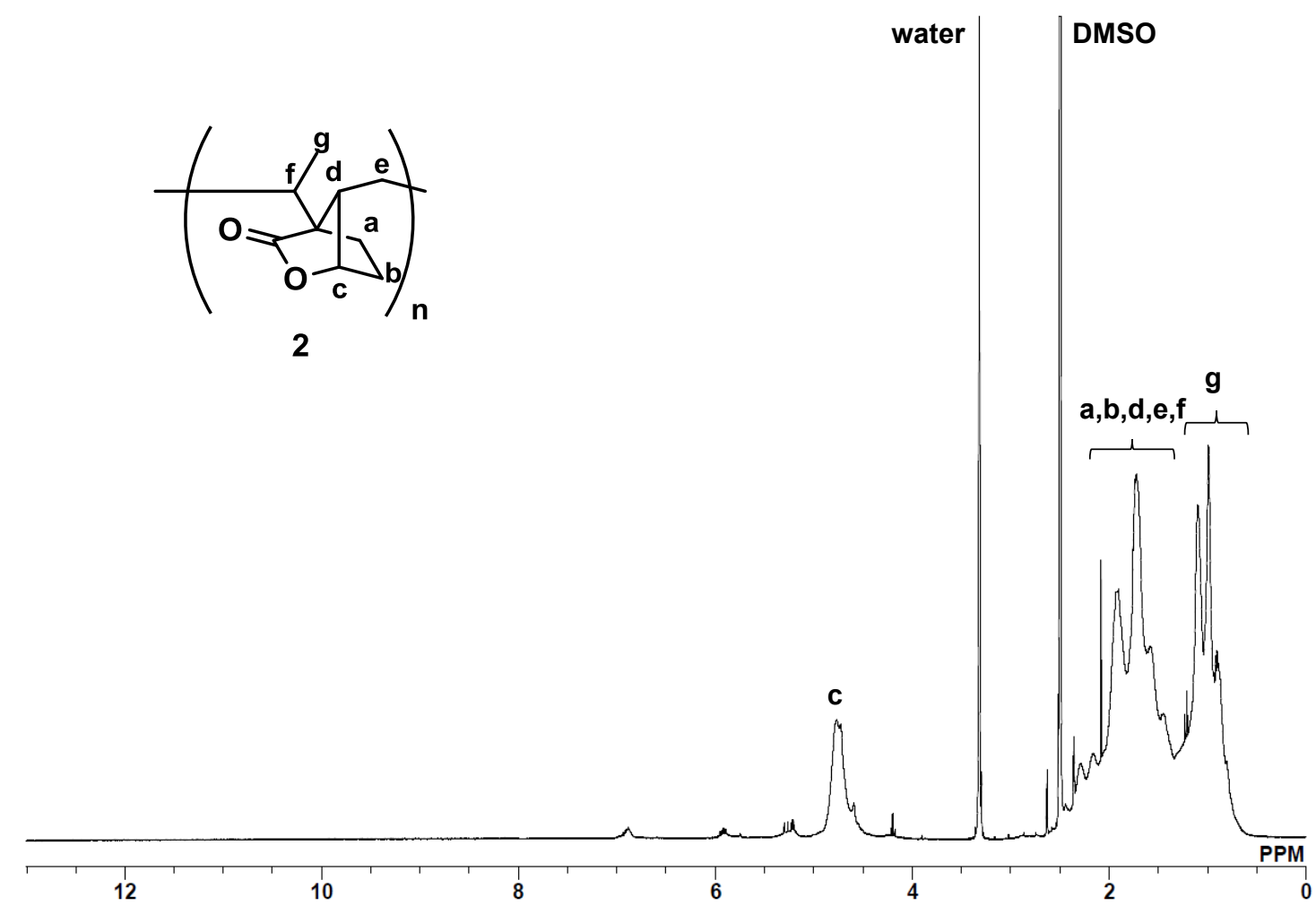

Figure S6. ${ }^{1} \mathrm{H}$ NMR spectrum $\left(500 \mathrm{MHz}, \mathrm{DMSO}-d_{6}\right)$ of $\mathrm{CO}_{2} /$ butadiene copolymer 2 


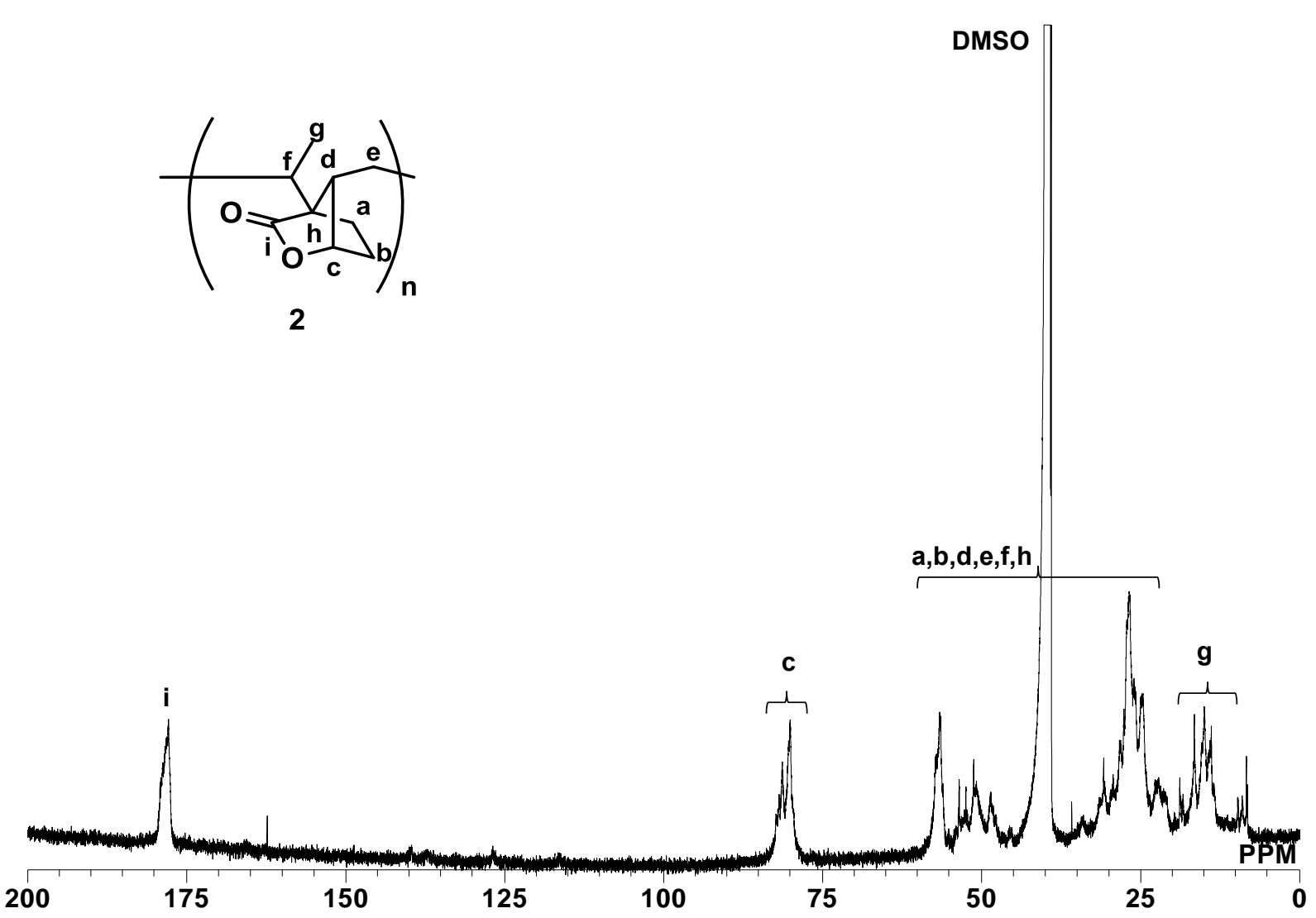

Figure S7. ${ }^{13} \mathrm{C}$ NMR spectrum $\left(126 \mathrm{MHz}, \mathrm{DMSO}-d_{6}\right)$ of $\mathrm{CO}_{2} /$ butadiene copolymer 2

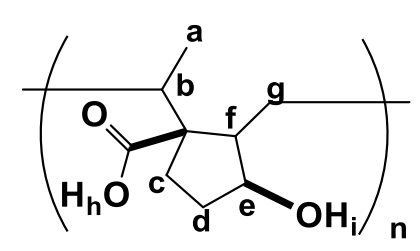

$3 a$

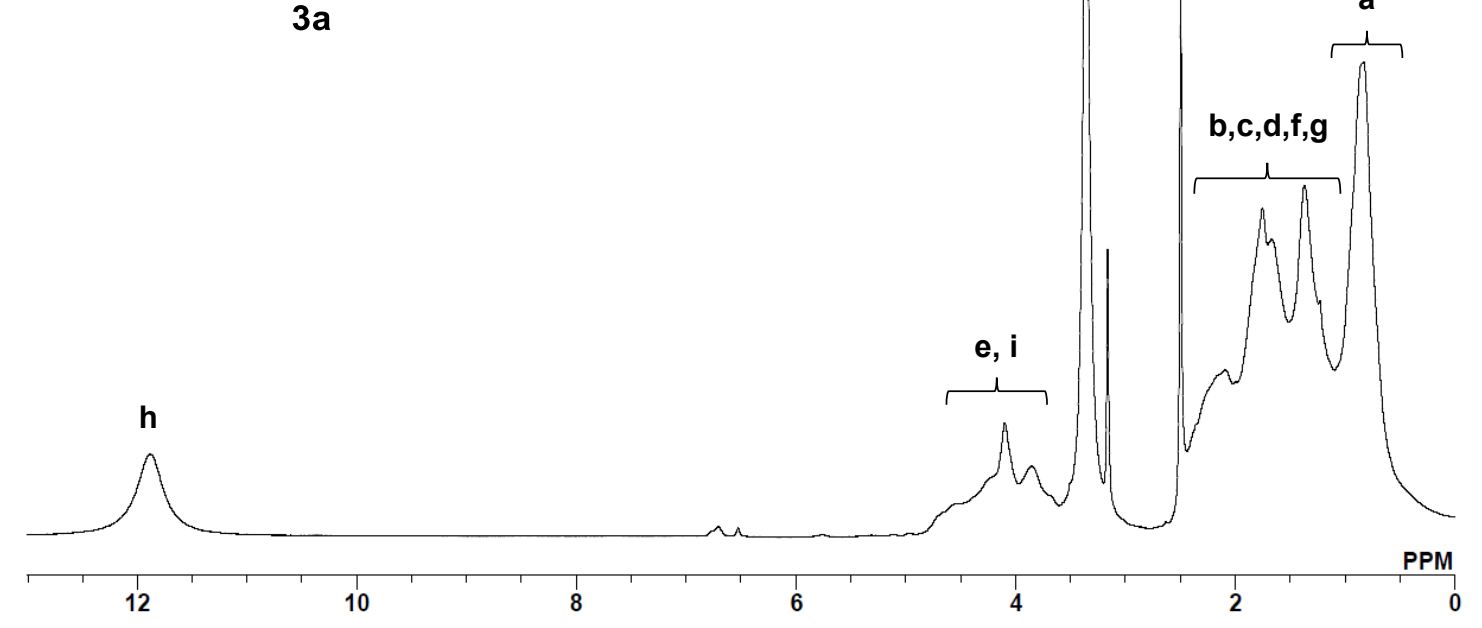

Figure S8. ${ }^{1} \mathrm{H}$ NMR spectrum $\left(500 \mathrm{MHz}, \mathrm{DMSO}-d_{6}\right)$ of hydrolysis product 3a 


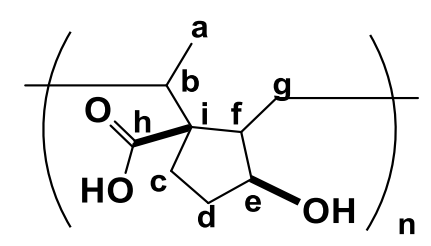

3a

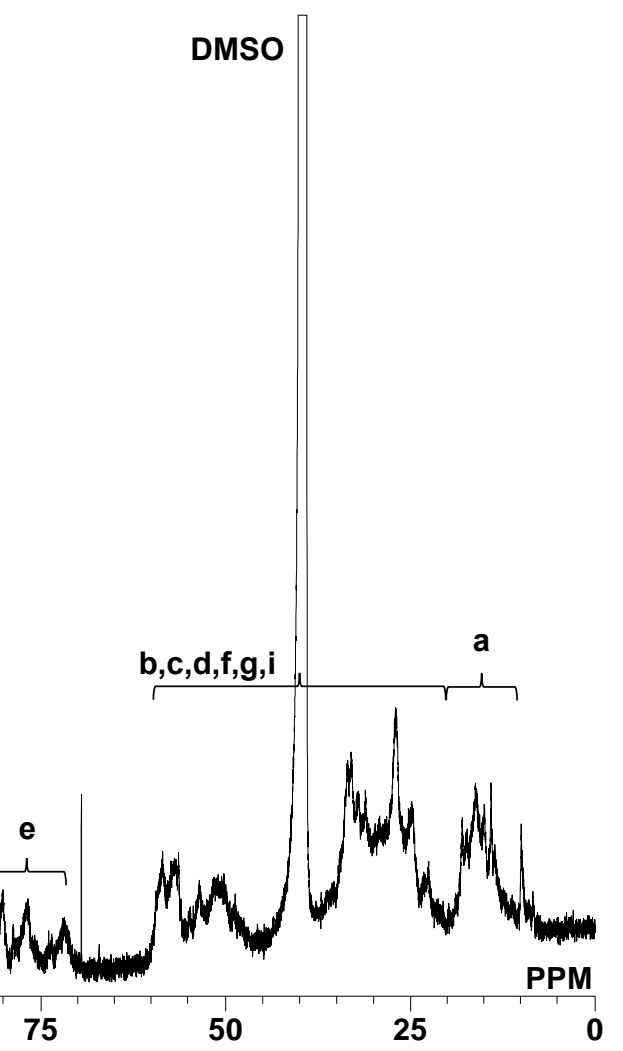

Figure S9. ${ }^{13} \mathrm{C}$ NMR spectrum $\left(126 \mathrm{MHz}\right.$, DMSO- $\left.d_{6}\right)$ of hydrolysis product 3a

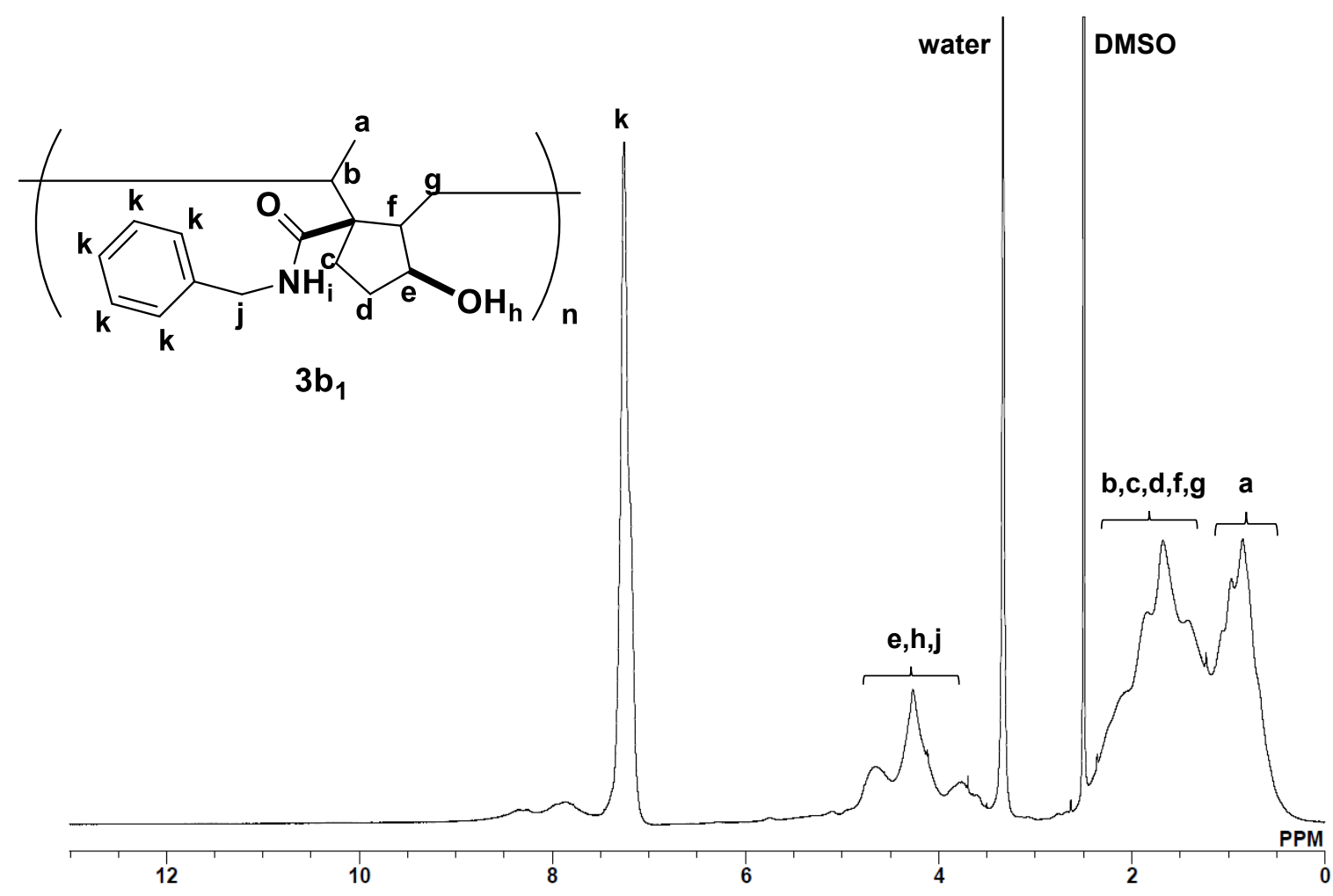

Figure S10. ${ }^{1} \mathrm{H}$ NMR spectrum $\left(500 \mathrm{MHz}\right.$, DMSO- $\left.d_{6}\right)$ of aminolysis product $\mathbf{3 b}_{1}$ 


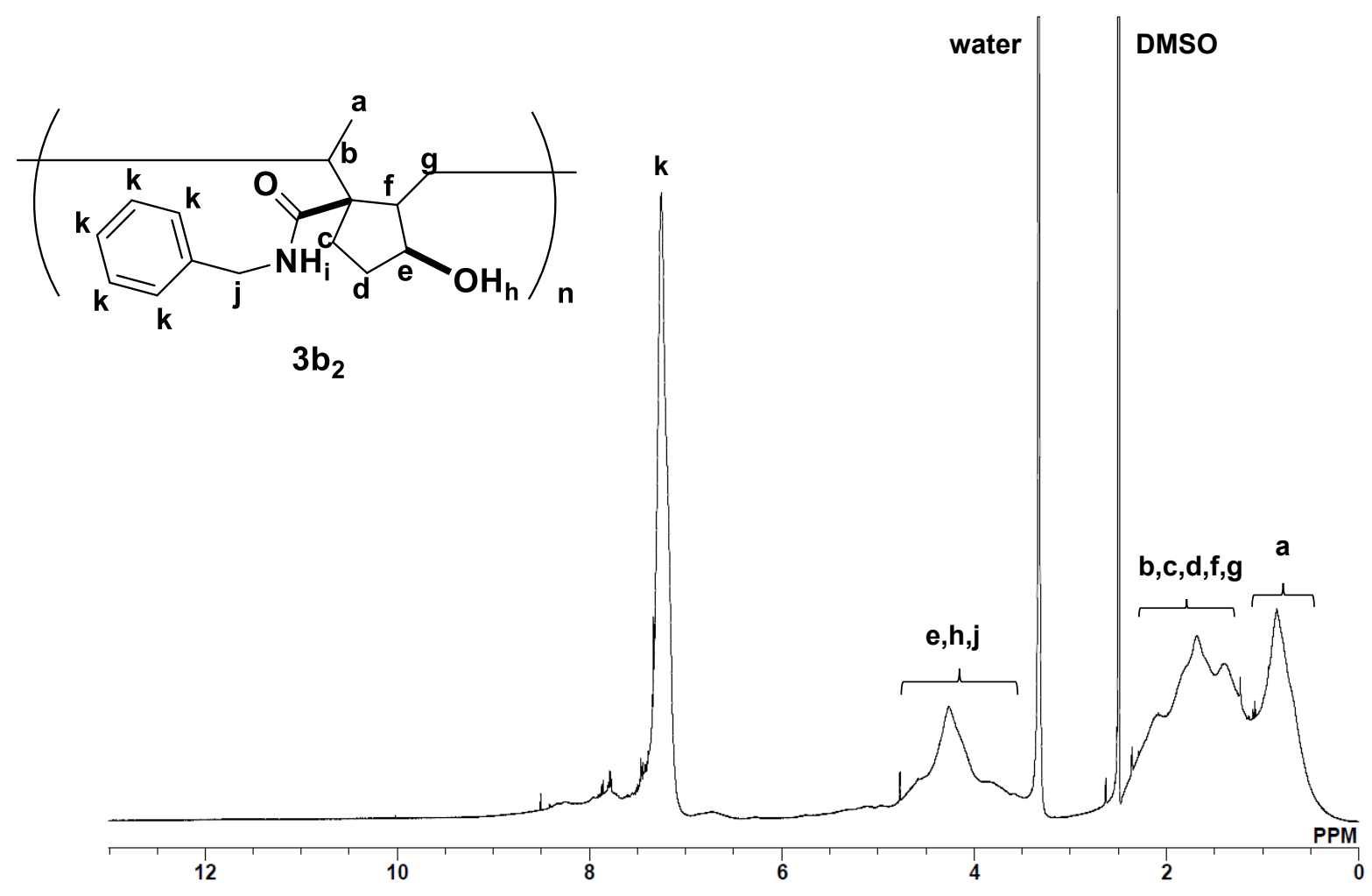

Figure S11. ${ }^{1} \mathrm{H}$ NMR spectrum $\left(500 \mathrm{MHz}, \mathrm{DMSO}-d_{6}\right)$ of aminolysis product $\mathbf{3} \mathbf{b}_{2}$

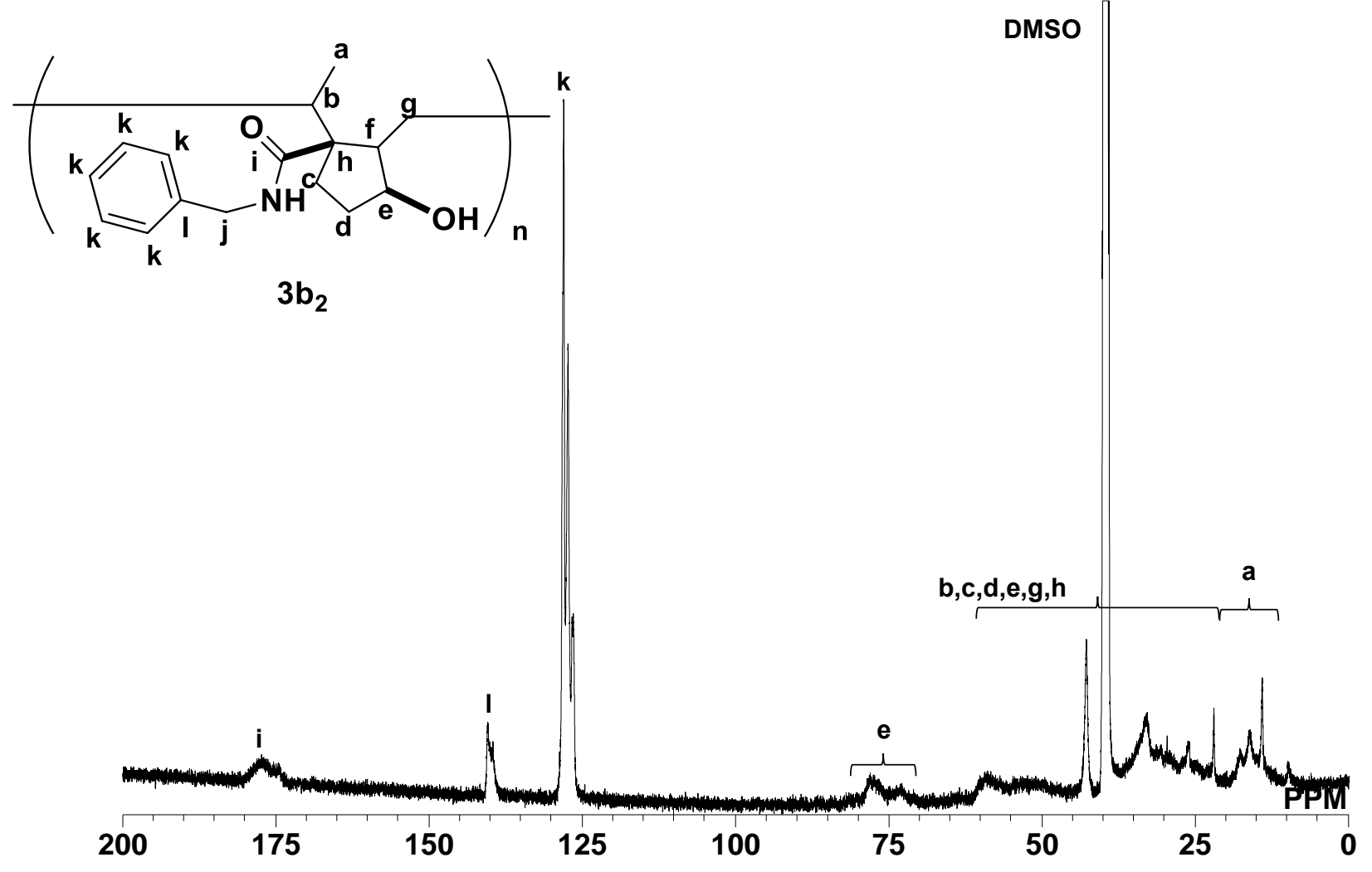

Figure S12. ${ }^{13} \mathrm{C}$ NMR spectrum $\left(126 \mathrm{MHz}\right.$, DMSO- $\left.d_{6}\right)$ of aminolysis product $\mathbf{3 b}_{2}$ 


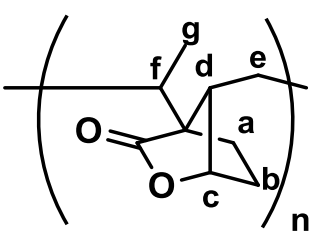

2'

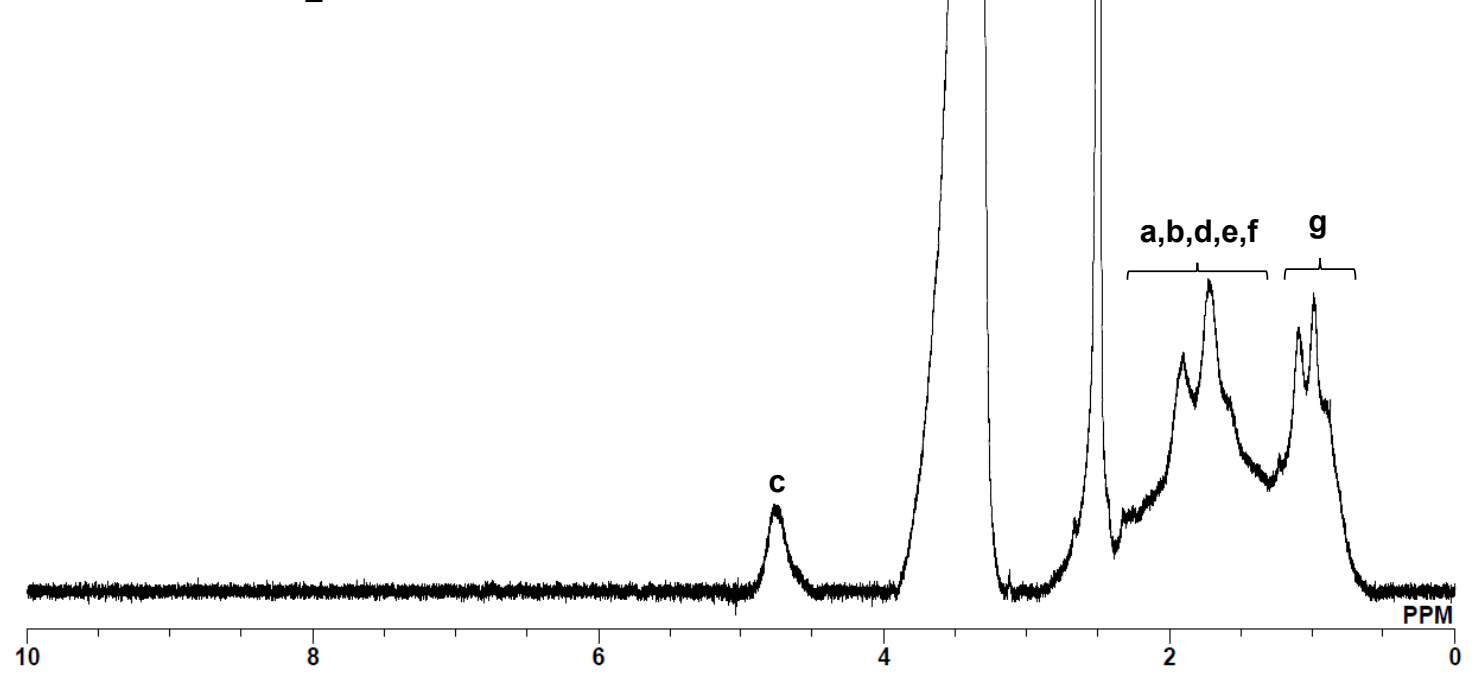

Figure S13. ${ }^{1} \mathrm{H}$ NMR spectrum (400 MHz, DMSO- $\left.d_{6}\right)$ of ring-closed polymer 2' (heated 3a)
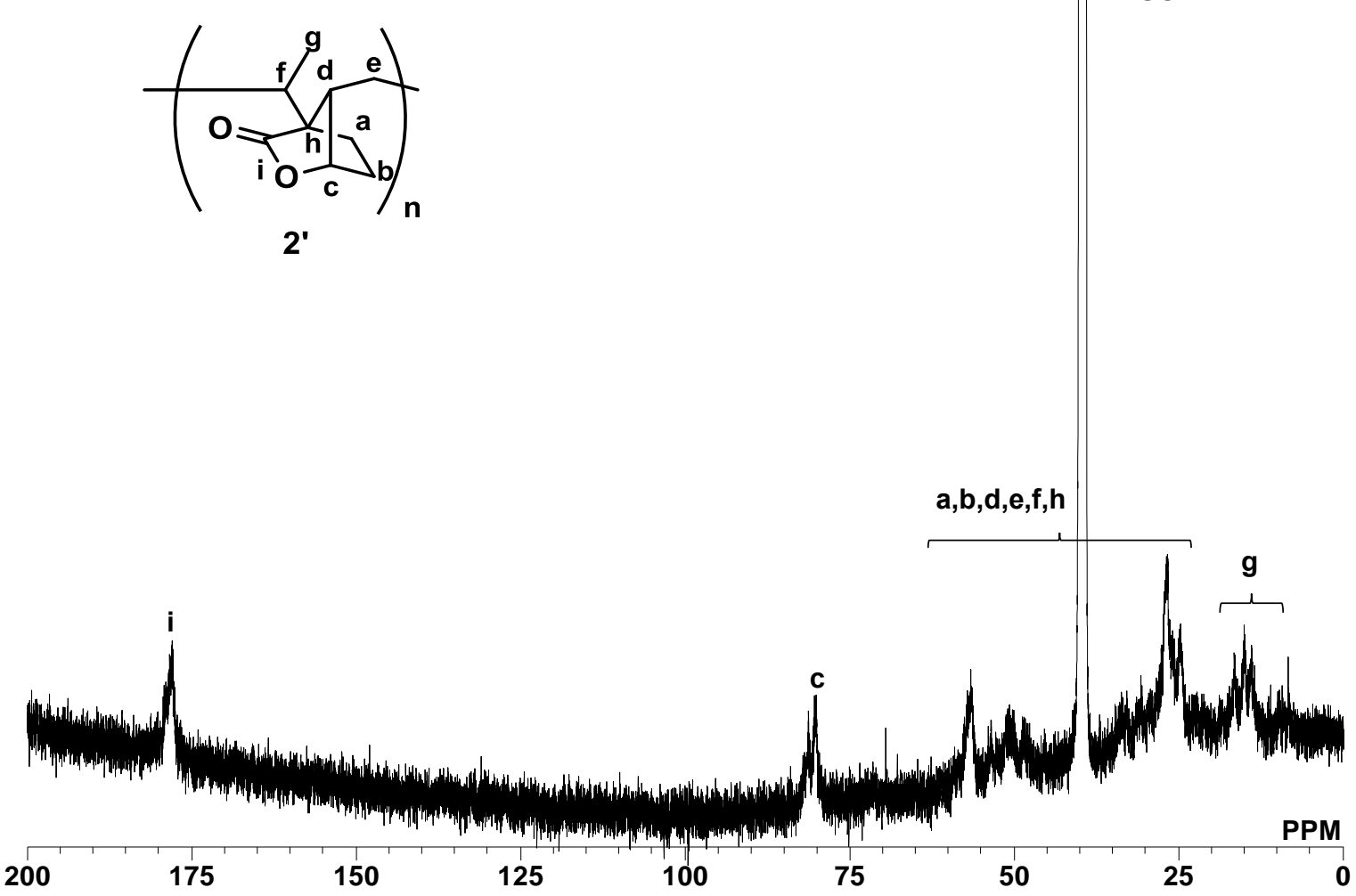

Figure S14. ${ }^{13} \mathrm{C}$ NMR spectrum (126 MHz, DMSO- $d_{6}$ ) of ring-closed polymer 2' (heated 3a) 


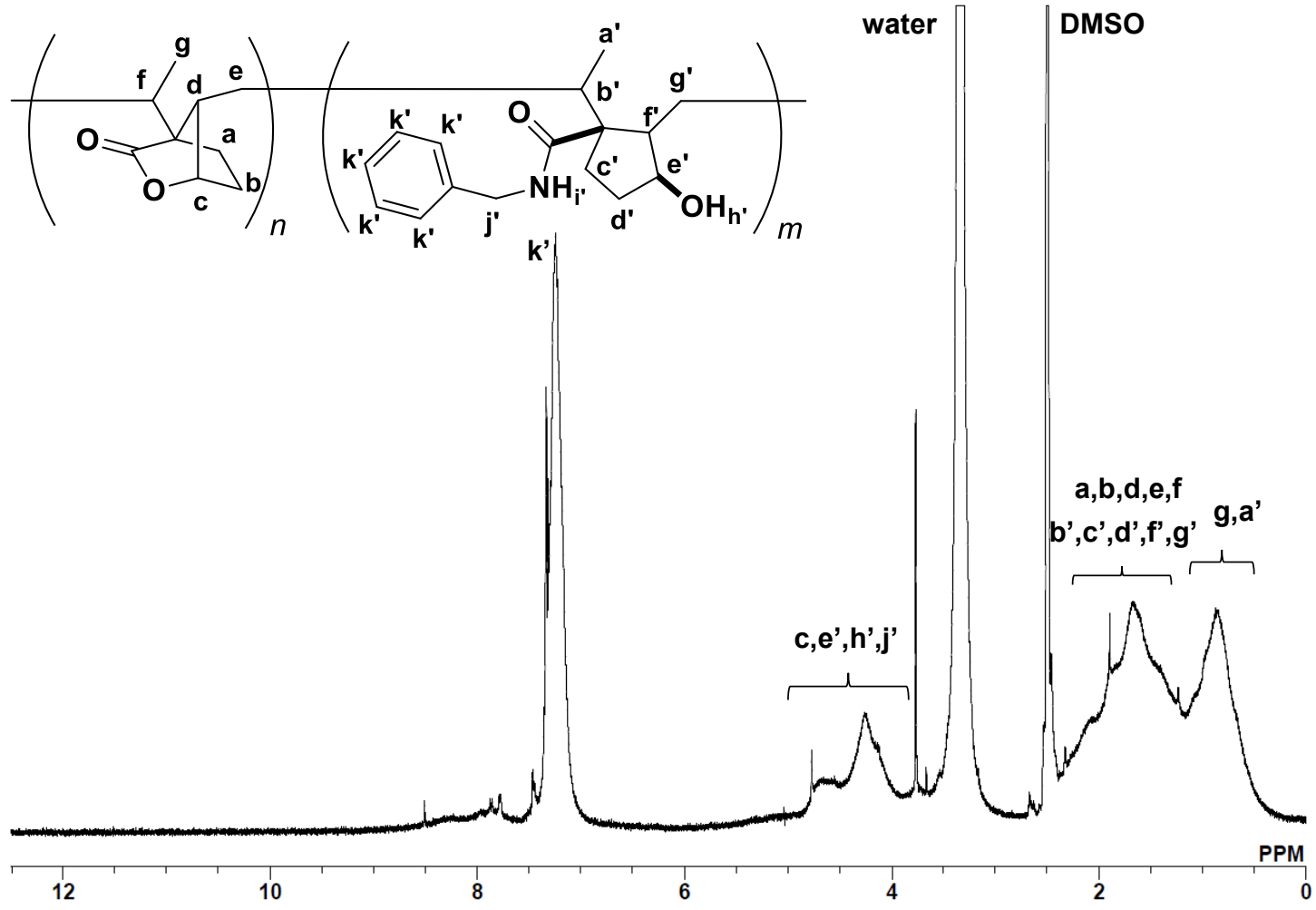

Figure S15. ${ }^{1} \mathrm{H}$ NMR spectrum ( $400 \mathrm{MHz}$, DMSO- $d_{6}$ ) of $\mathbf{3 b}_{2}$ after $45 \mathrm{~min}$. heating at $180{ }^{\circ} \mathrm{C}$
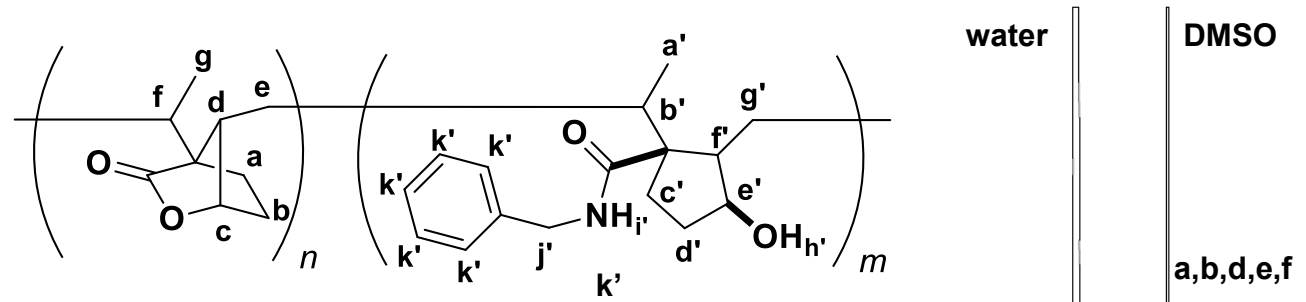

$4 b_{1}$

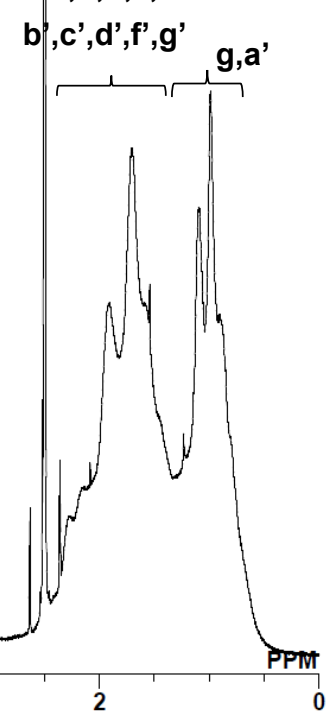

Figure S16. ${ }^{1} \mathrm{H}$ NMR spectrum $\left(500 \mathrm{MHz}\right.$, DMSO- $\left.d_{6}\right)$ of $\mathbf{3 b}_{1}$ after $60 \mathrm{~min}$. heating at $260{ }^{\circ} \mathrm{C}$ 


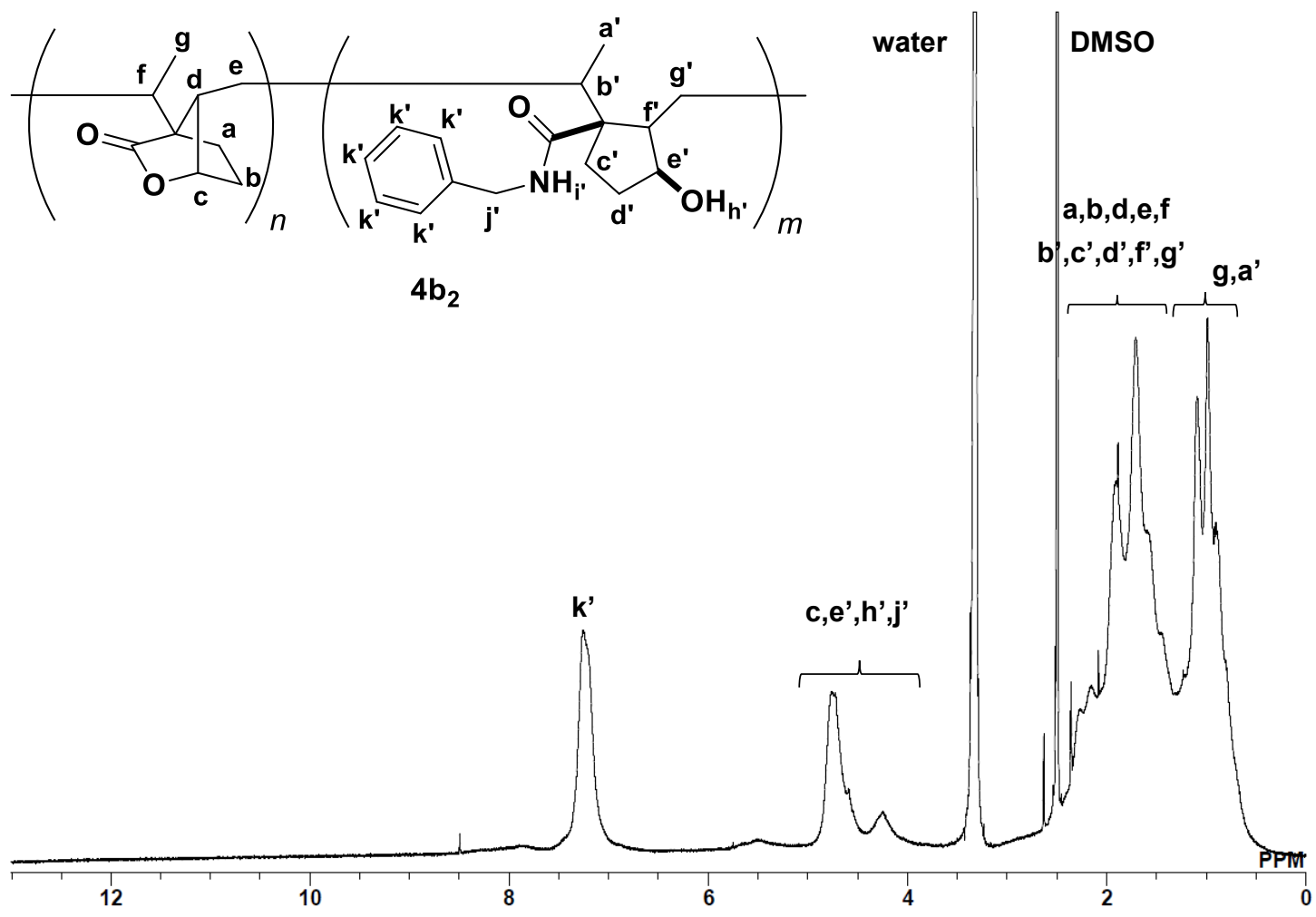

Figure S17. ${ }^{1} \mathrm{H}$ NMR spectrum $\left(500 \mathrm{MHz}, \mathrm{DMSO}-d_{6}\right)$ of $\mathbf{3} \mathbf{b}_{2}$ after $60 \mathrm{~min}$. heating at $260{ }^{\circ} \mathrm{C}$
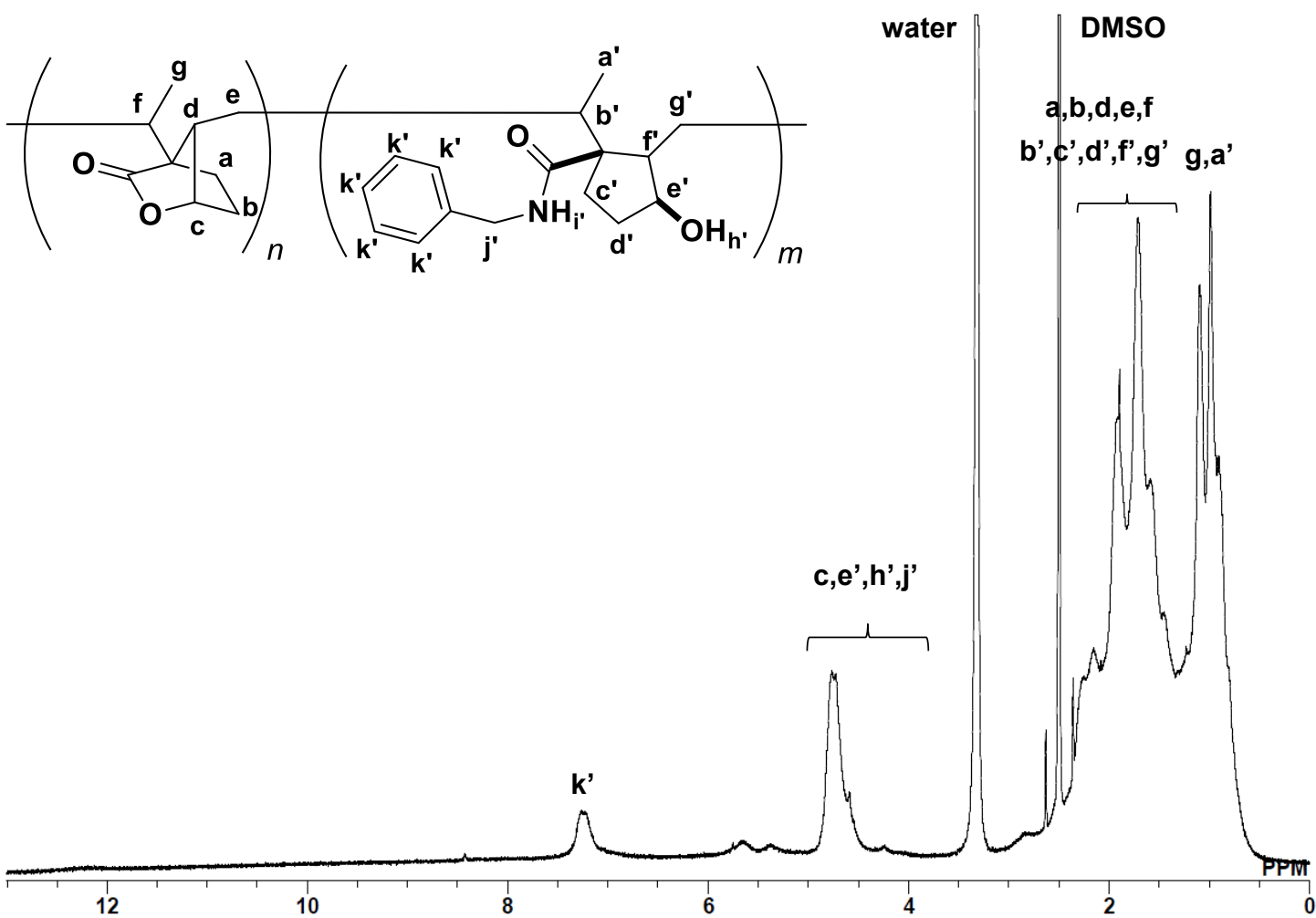

Figure S18. ${ }^{1} \mathrm{H}$ NMR spectrum $\left(500 \mathrm{MHz}, \mathrm{DMSO}-d_{6}\right)$ of $\mathbf{3 b}_{\mathbf{1}}$ after TG measurement 


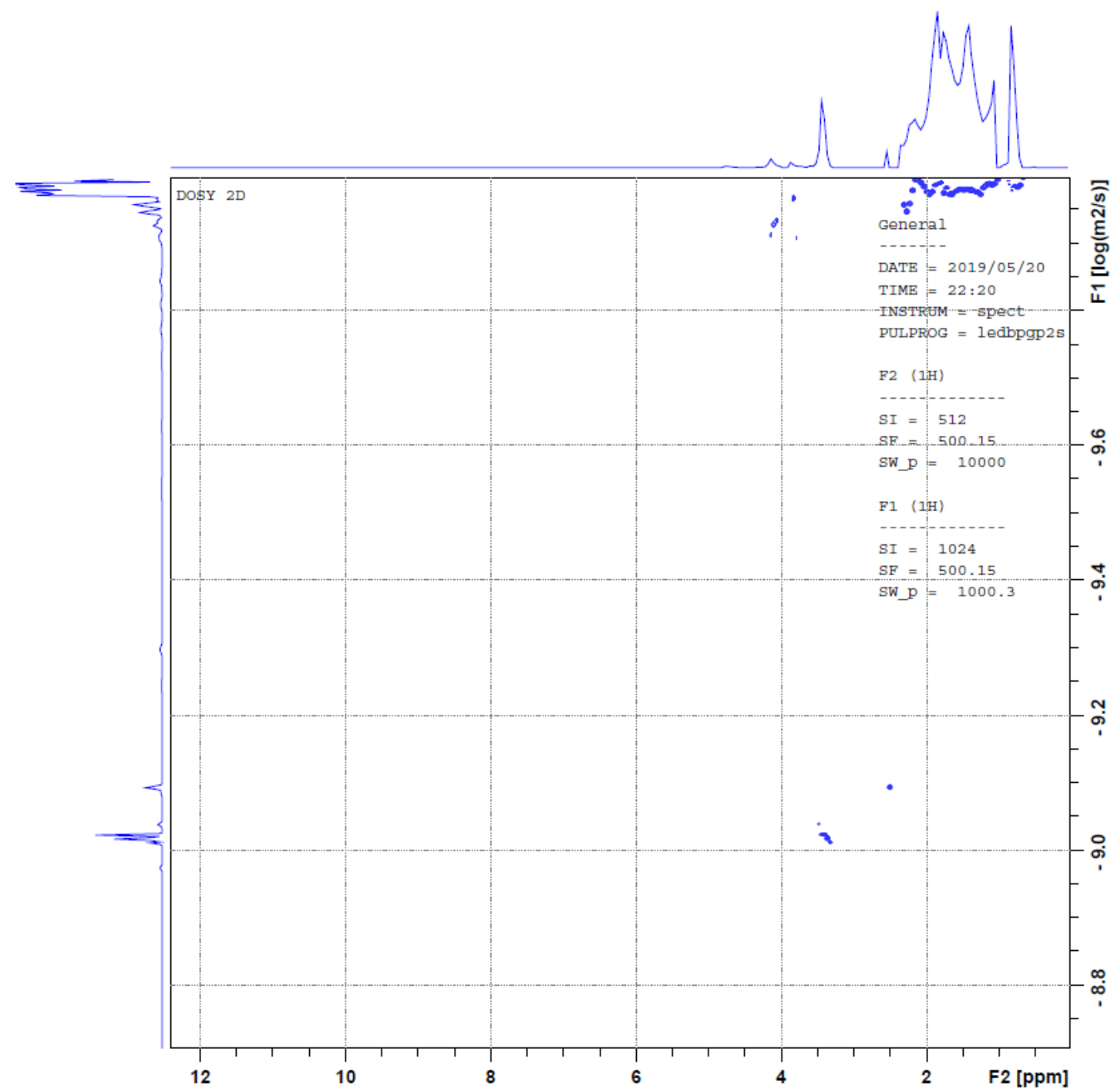

Figure S19. DOSY NMR spectrum (500 MHz, DMSO- $d_{6}$ ) of hydrolysis product (3a) 

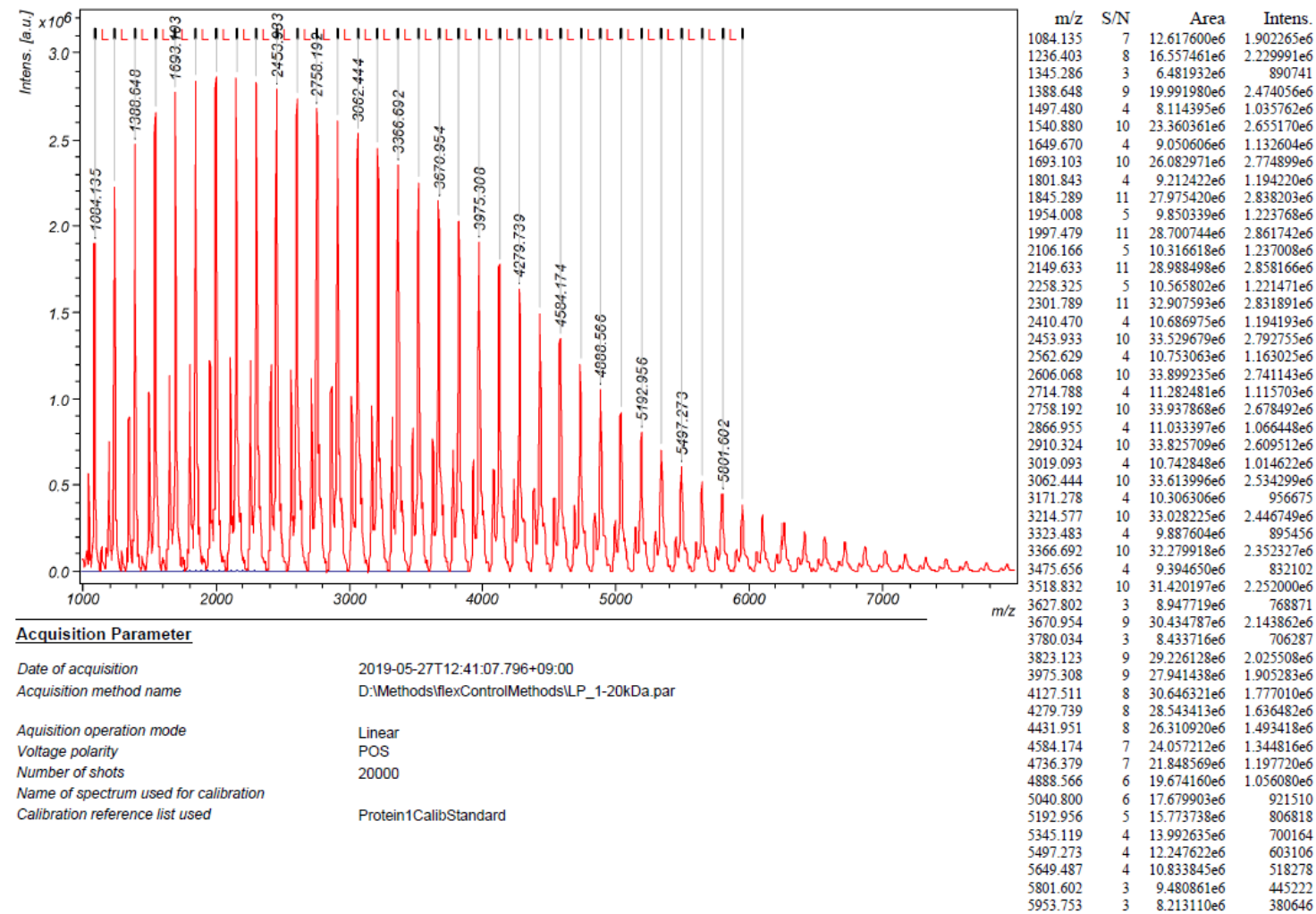

Figure S20. MALDI-TOF MS spectrum of $\mathrm{CO}_{2} /$ butadiene copolymer 2 (Interval L $=152$ was detected.)

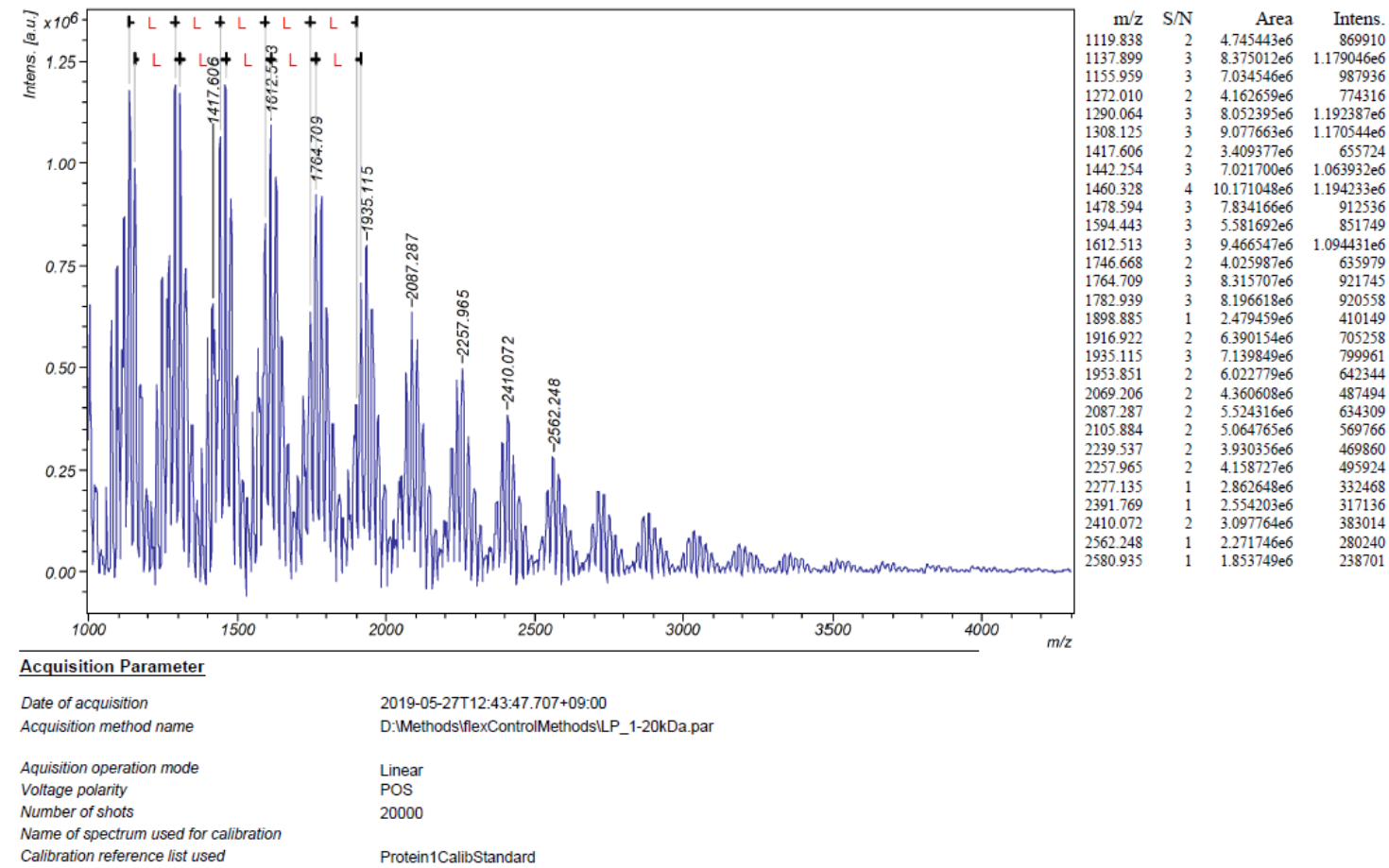

Figure S21. MALDI-TOF MS spectrum of hydrolysis product 3a

(Interval L $=152$ (not 170) was detected. Dehydration upon laser irradiation is suspected.) 


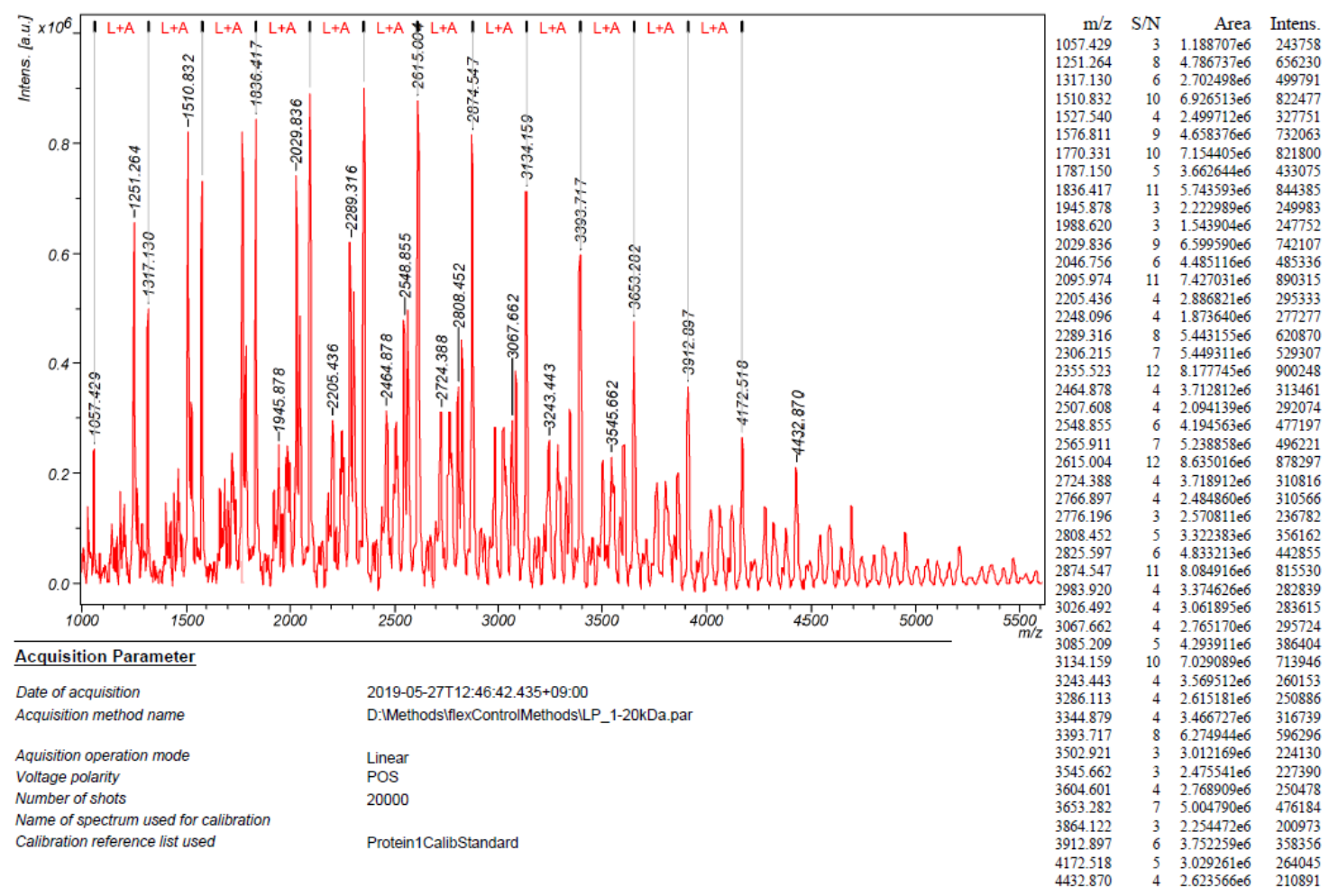

Figure S22. MALDI-TOF MS spectrum of aminolysis product $\mathbf{3} \mathbf{b}_{\mathbf{2}}$ (Interval $\mathrm{L}+\mathrm{A}=259$ was detected.)
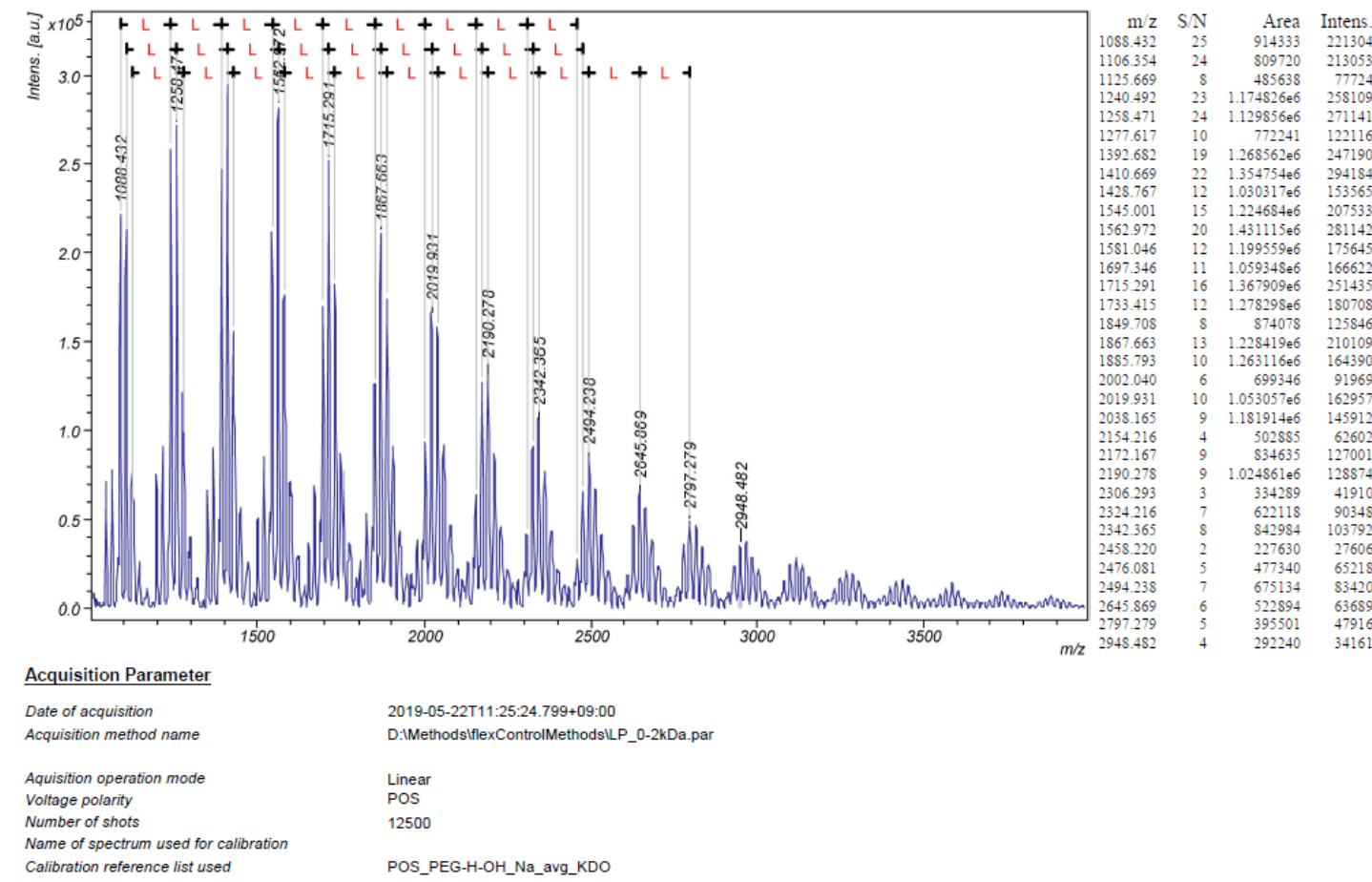

Figure S23. MALDI-TOF MS spectrum of ring-closed polymer 2' (Interval L = 152 was detected.) 


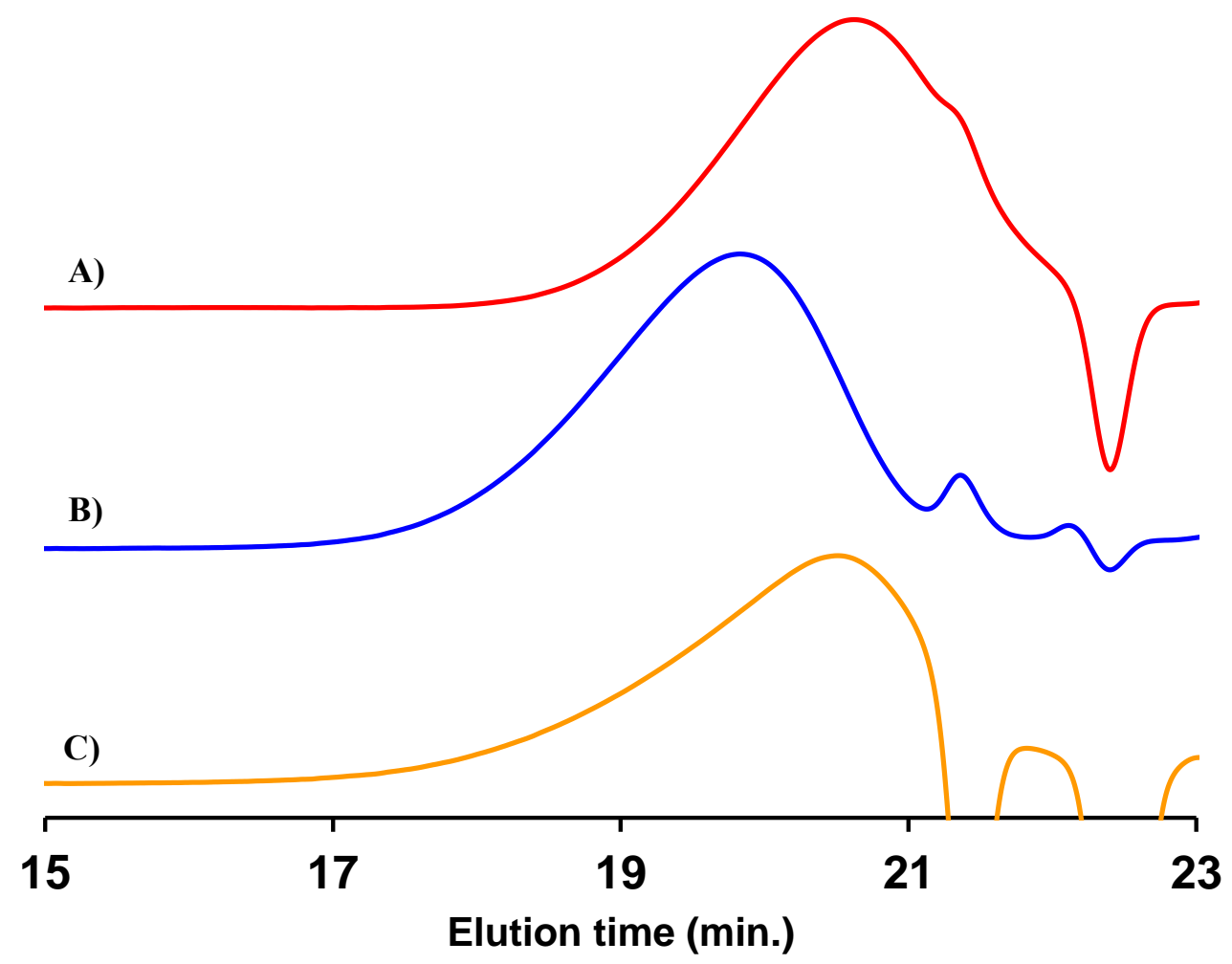

Figure S24. SEC charts of the $\mathrm{CO}_{2} /$ butadiene copolymer $\mathbf{2}(\mathrm{A})$, aminolysis product $\mathbf{3} \mathbf{b}_{2}$ (B) and $\mathbf{4} \mathbf{b}_{2}$ (C)

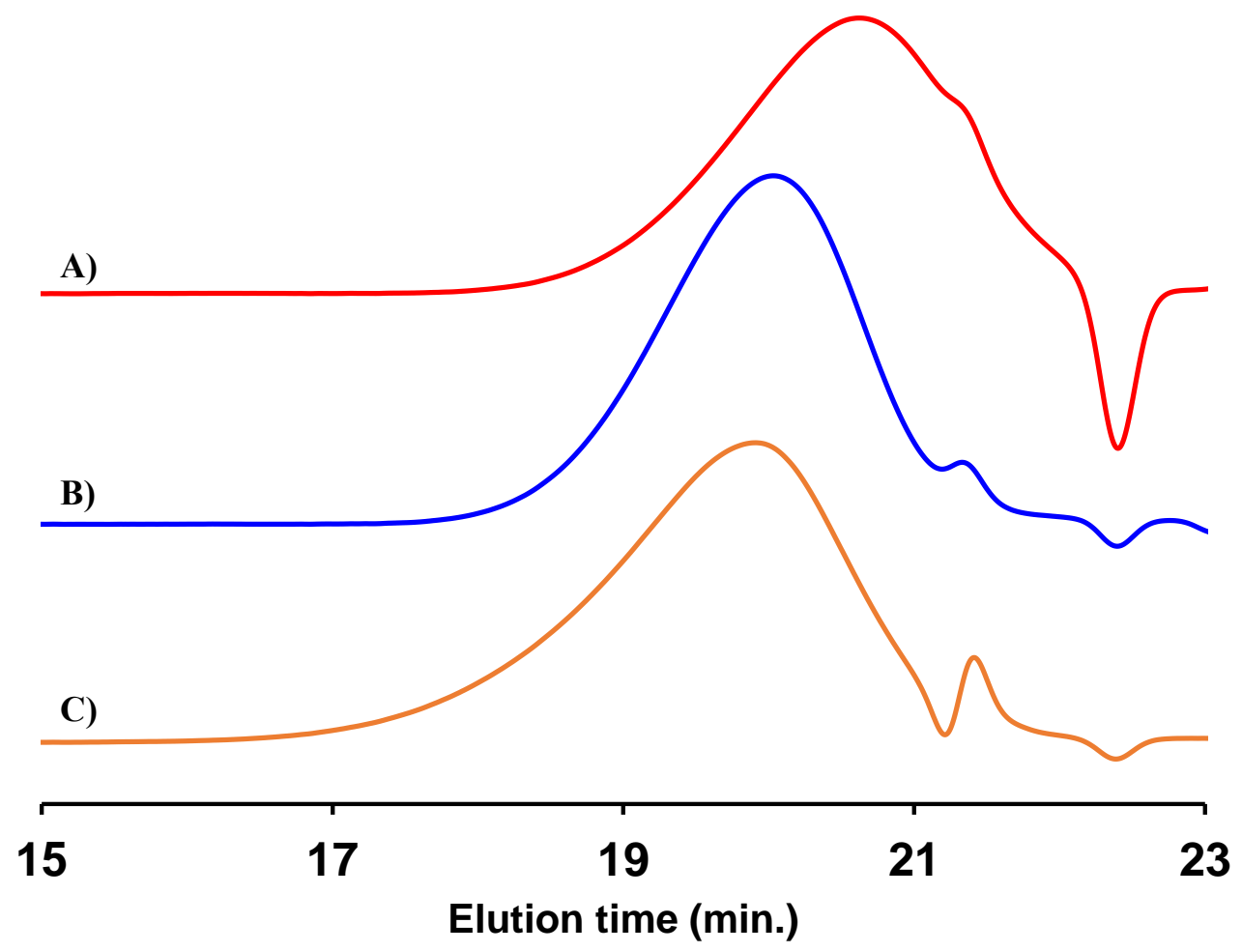

Figure S25. SEC charts of the $\mathrm{CO}_{2} /$ butadiene copolymer 2 (A), 55\% conversion aminolysis product $\mathbf{3} \mathbf{b}_{1}(\mathrm{~B})$, and ring-closed aminolysis product $\mathbf{5} \mathbf{b}_{\mathbf{1}}(\mathrm{C})$ 


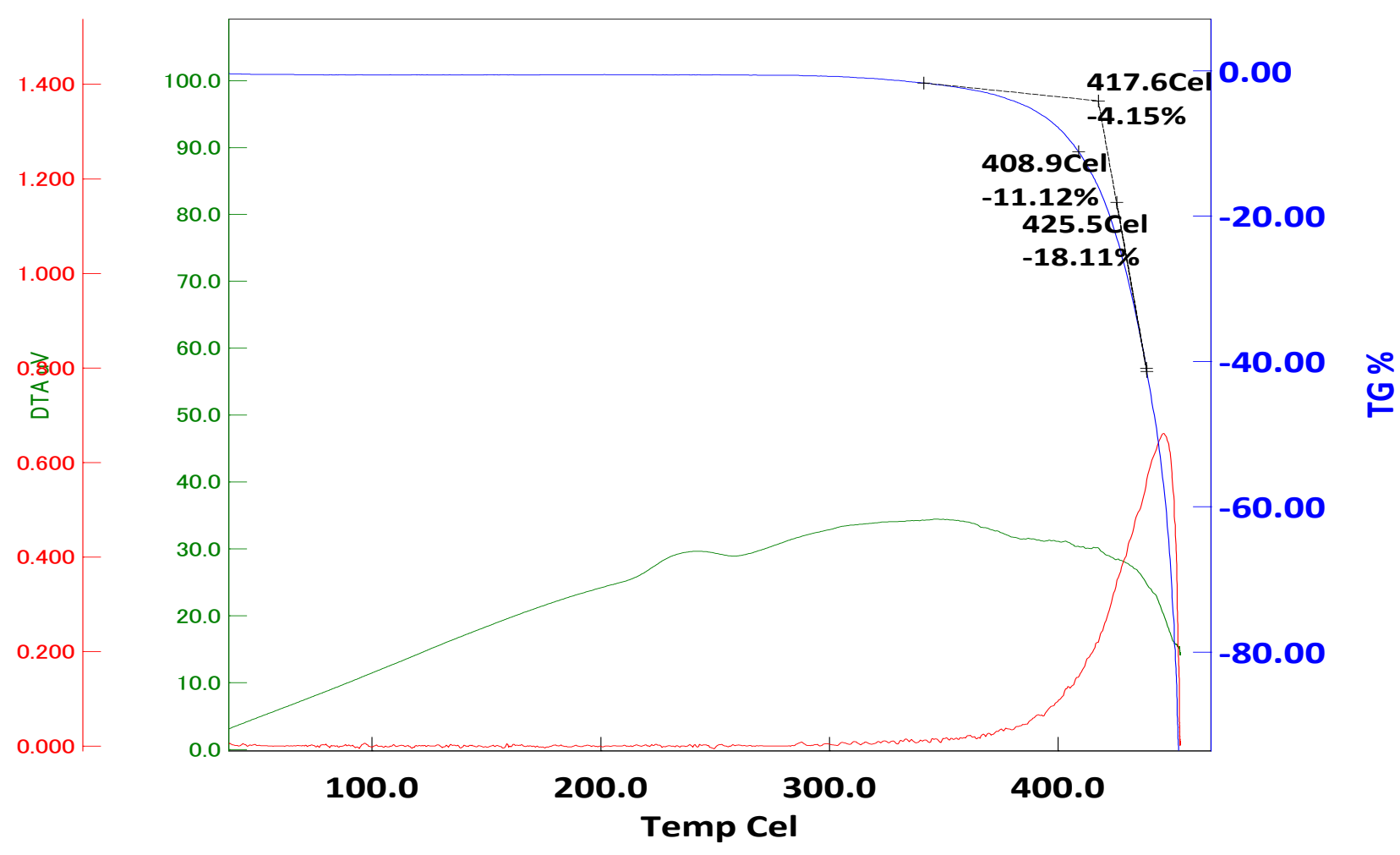

Figure S26. TG chart of $\mathrm{CO}_{2} /$ butadiene copolymer 2

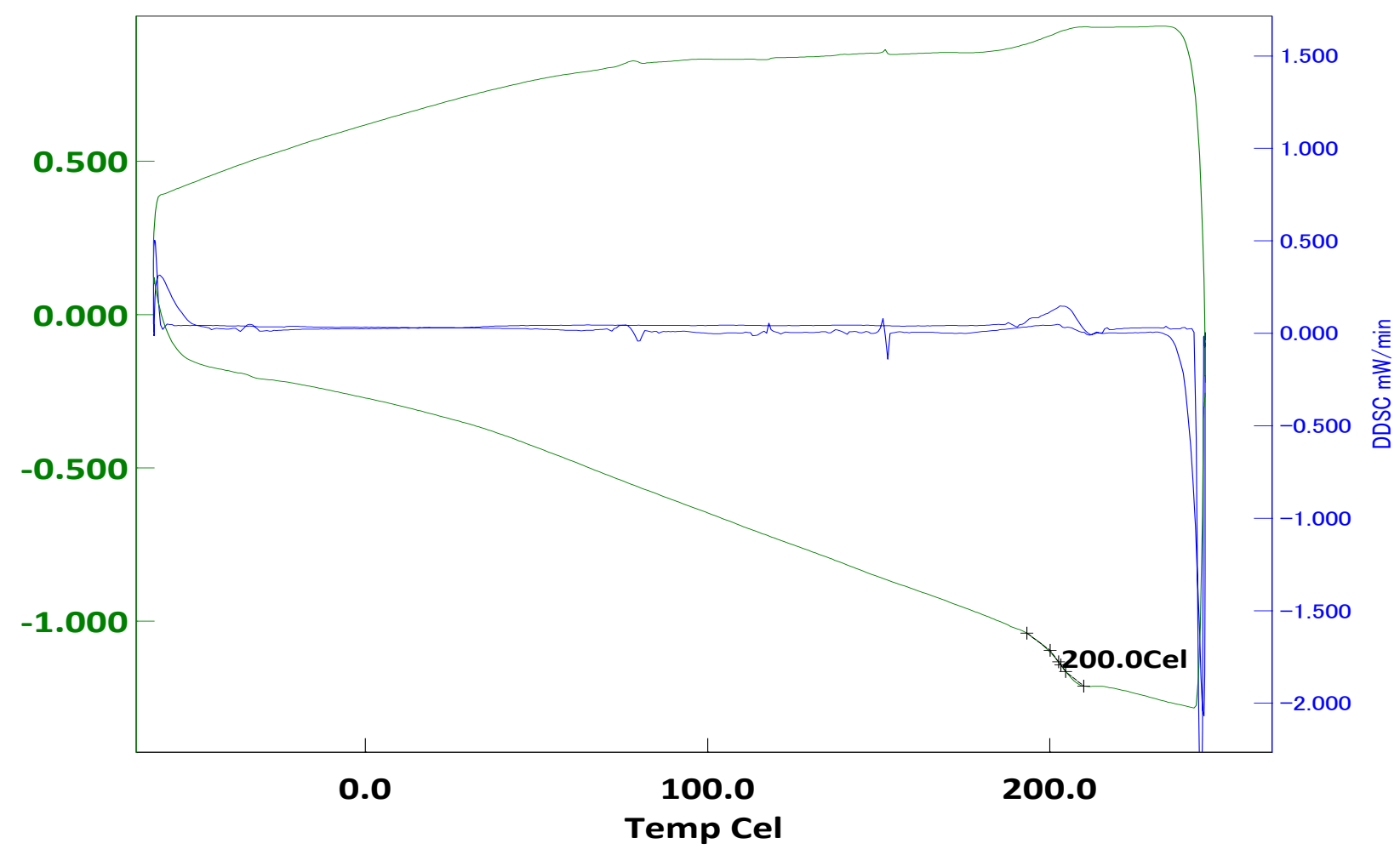

Figure S27. DSC chart of $\mathrm{CO}_{2} /$ butadiene copolymer 2 


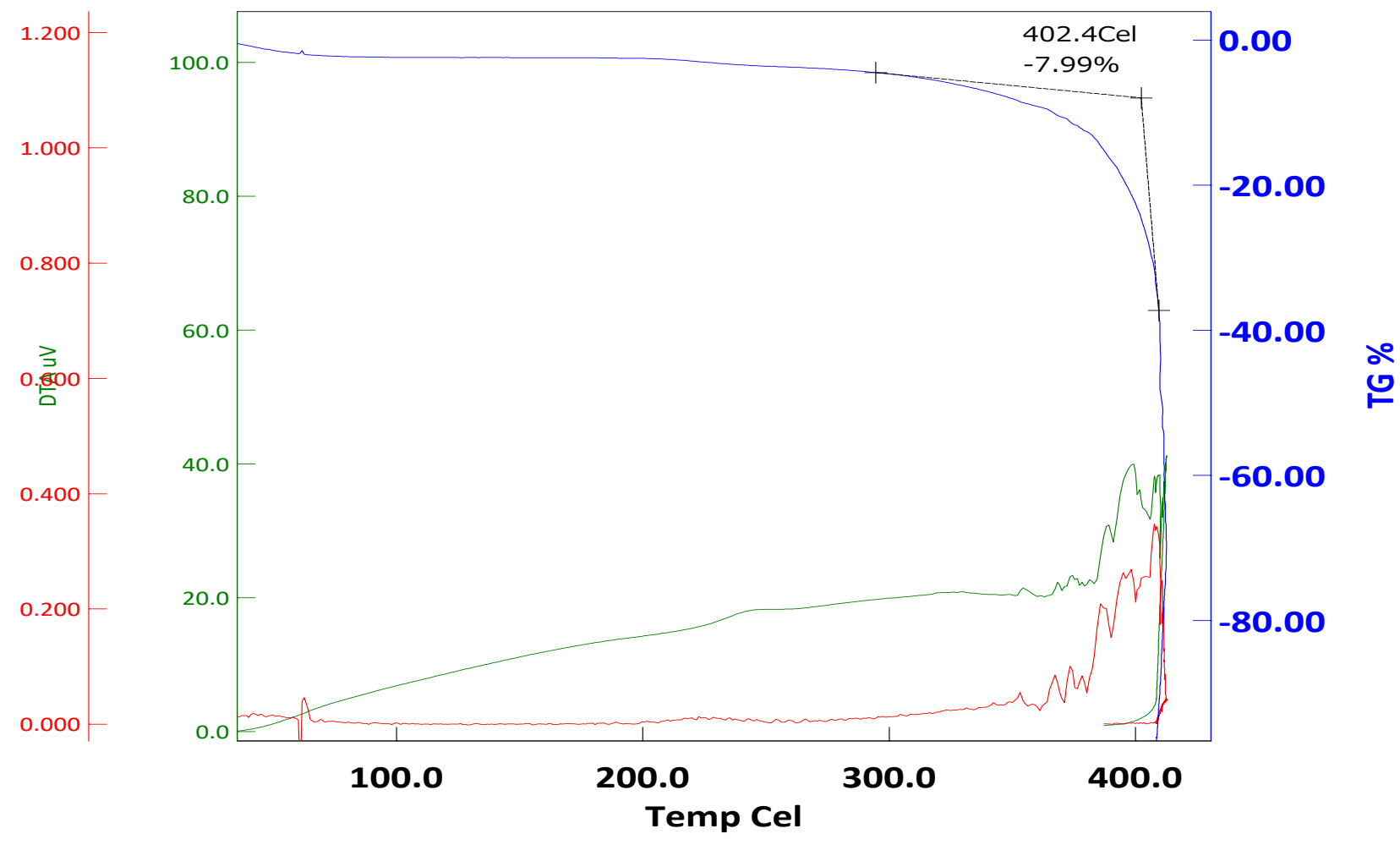

Figure S28. TG chart of ring-closed polymer 2' (heated 3a)

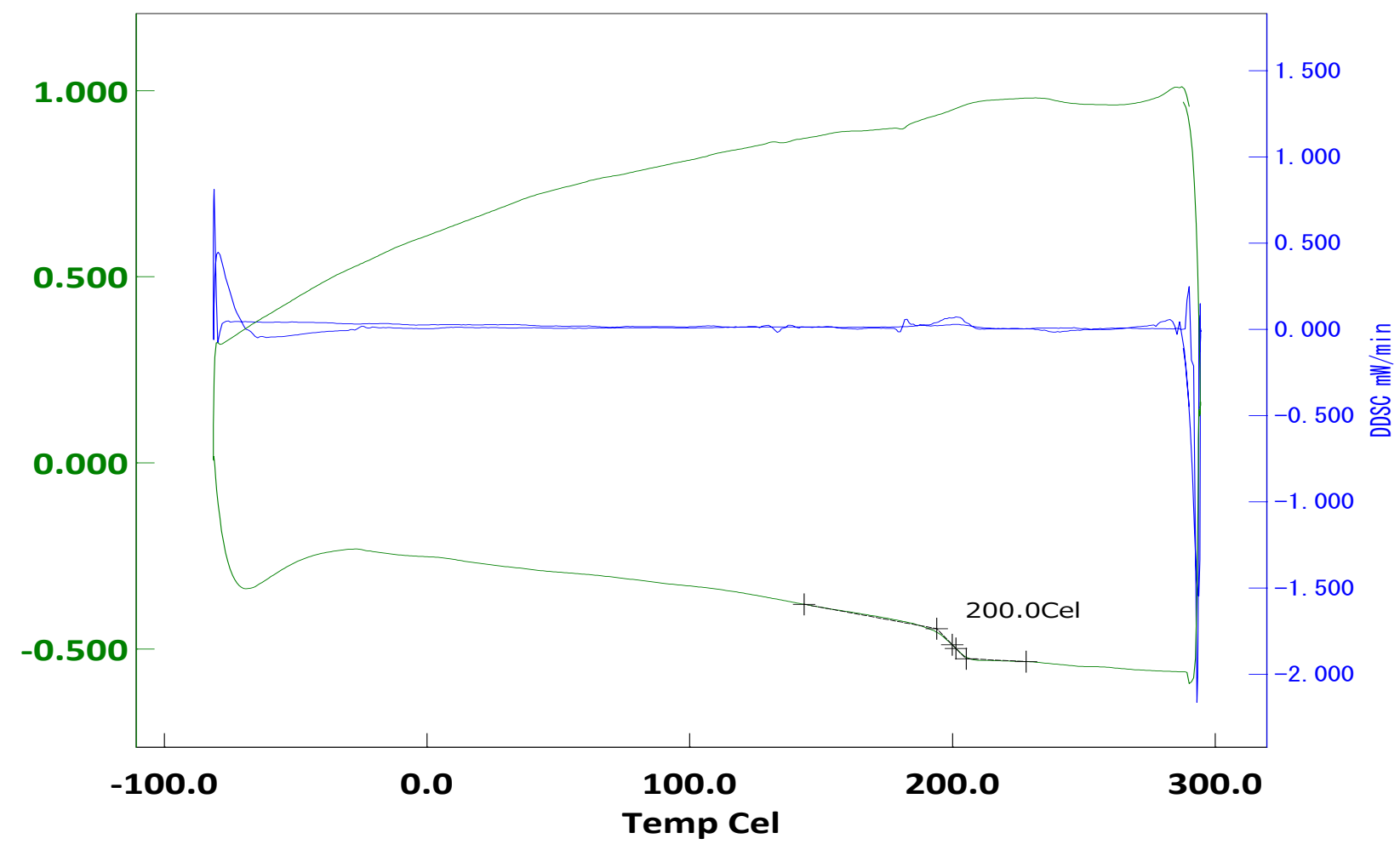

Figure S29. DSC chart of ring-closed polymer 2' (heated 3a) 


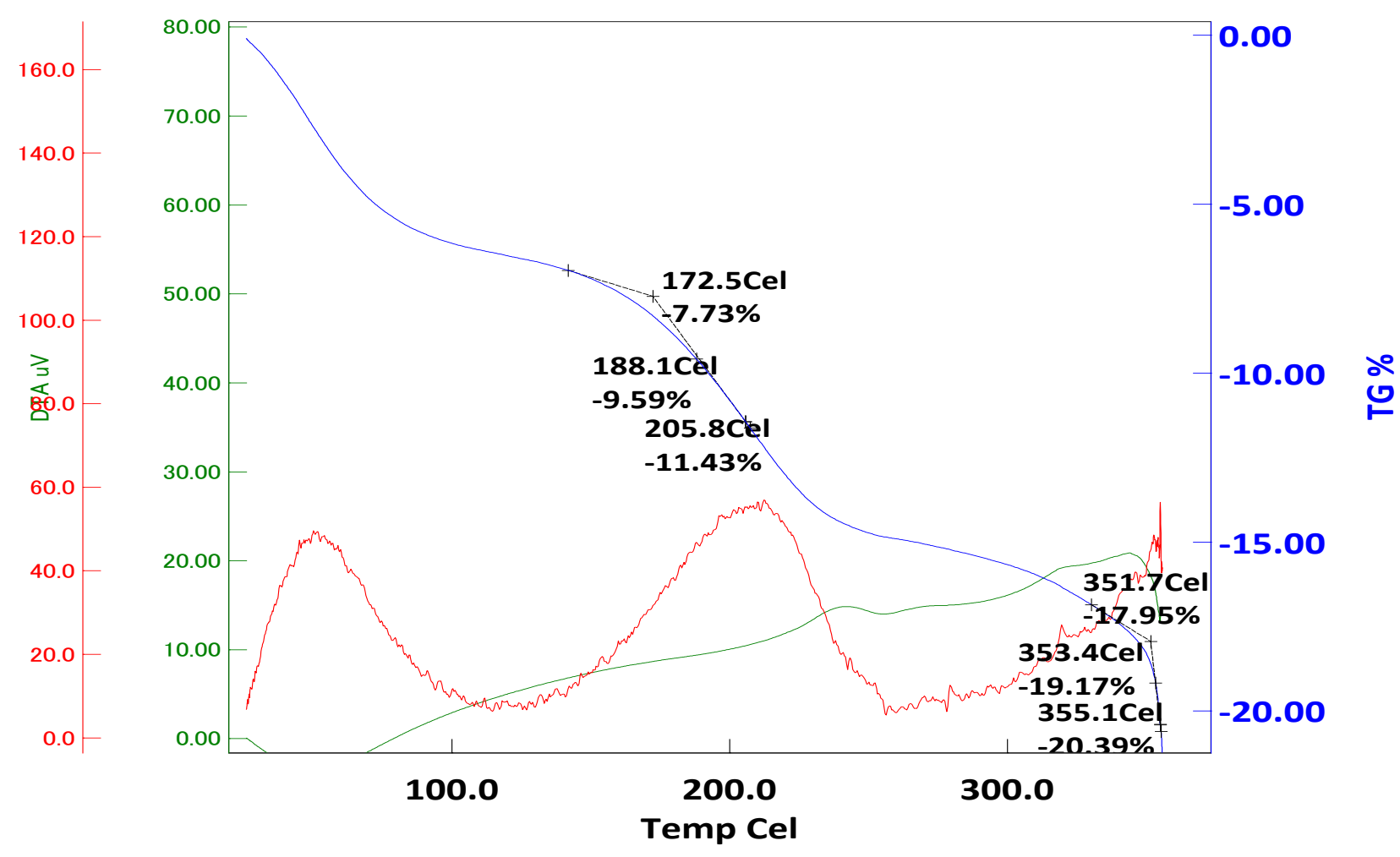

Figure S30. TG chart of hydrolysis product 3a

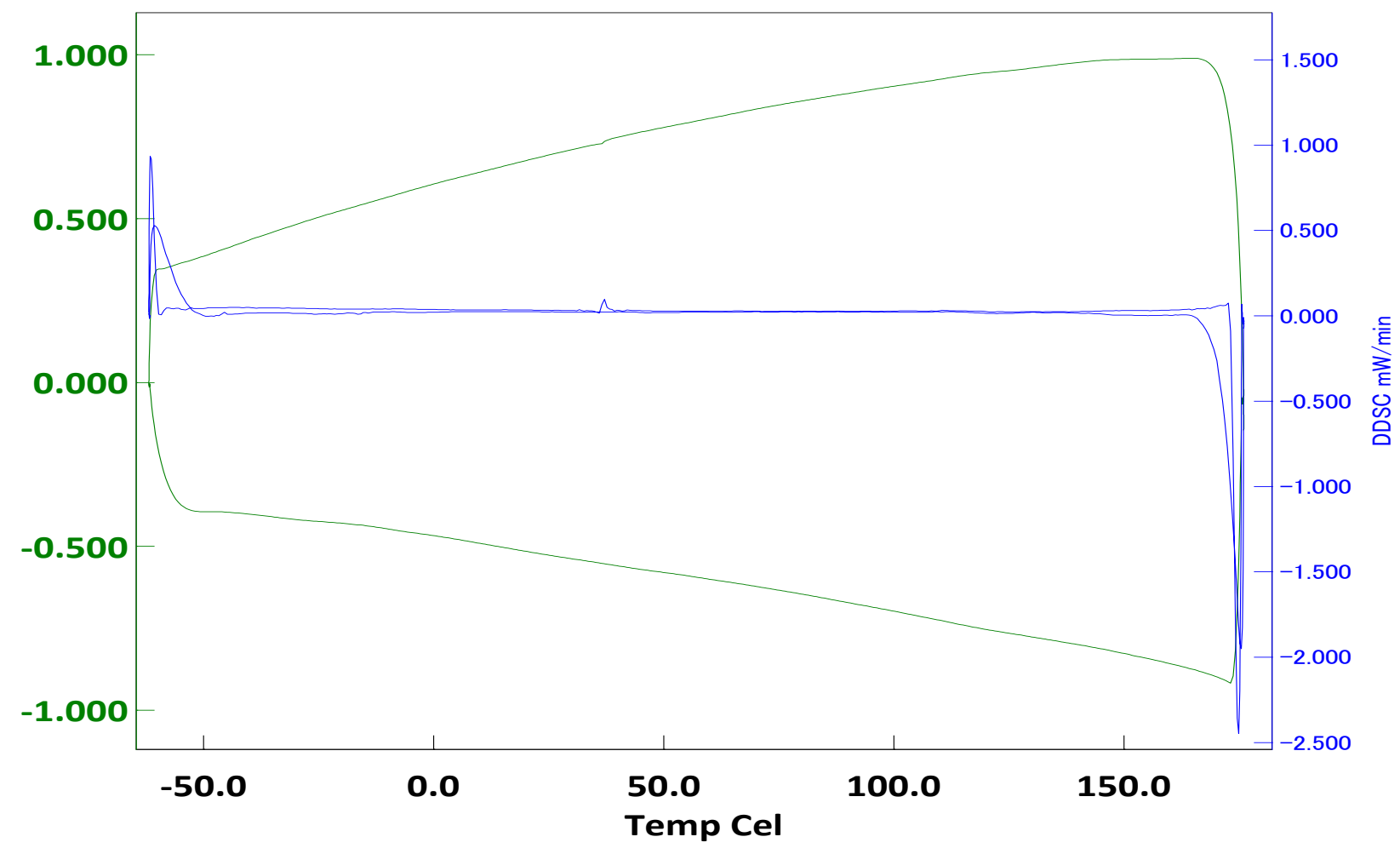

Figure S31. DSC chart of hydrolysis product 3a 


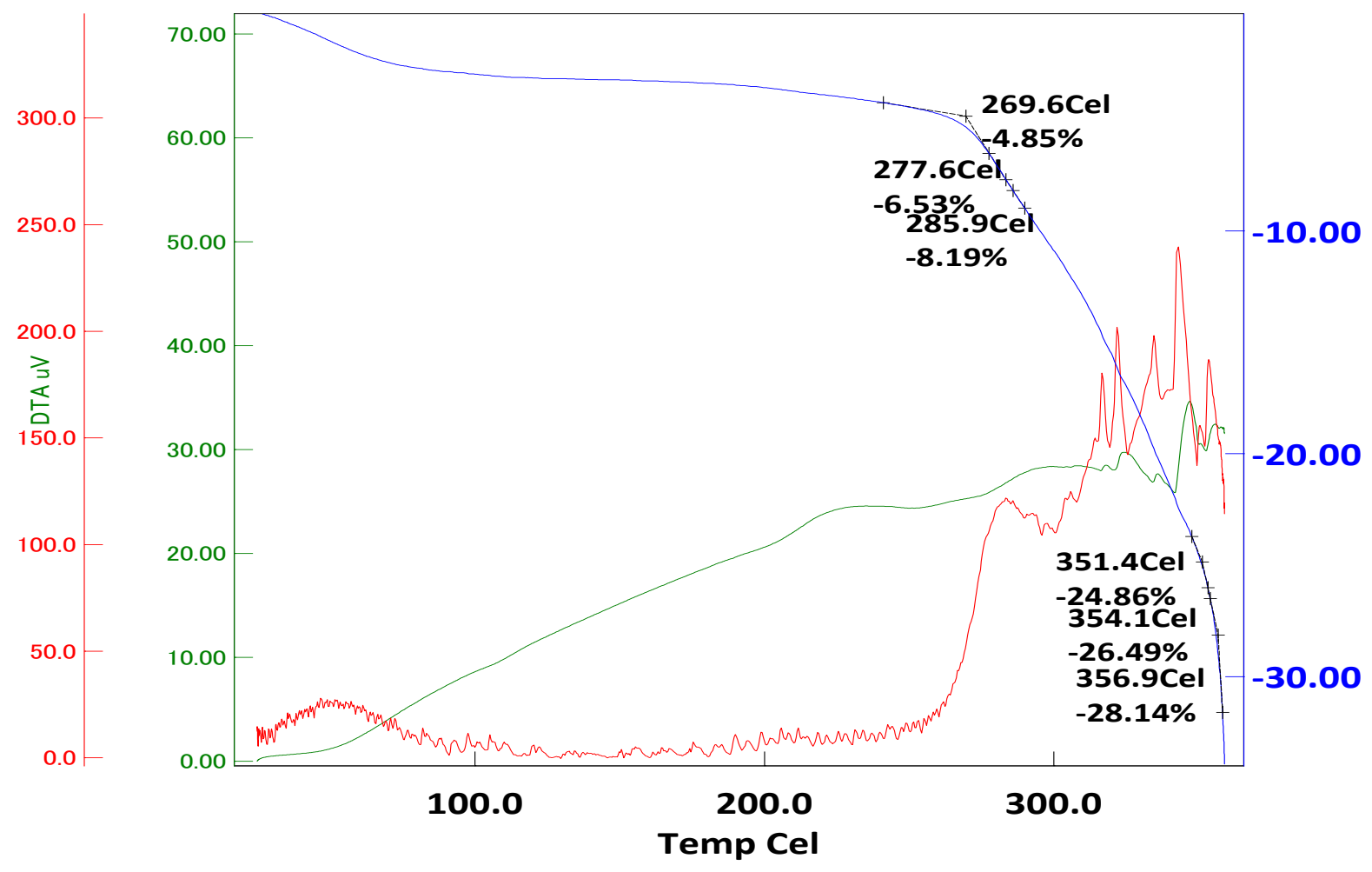

Figure S32. TG chart of aminolysis product $\mathbf{3} \mathbf{b}_{\mathbf{1}}$

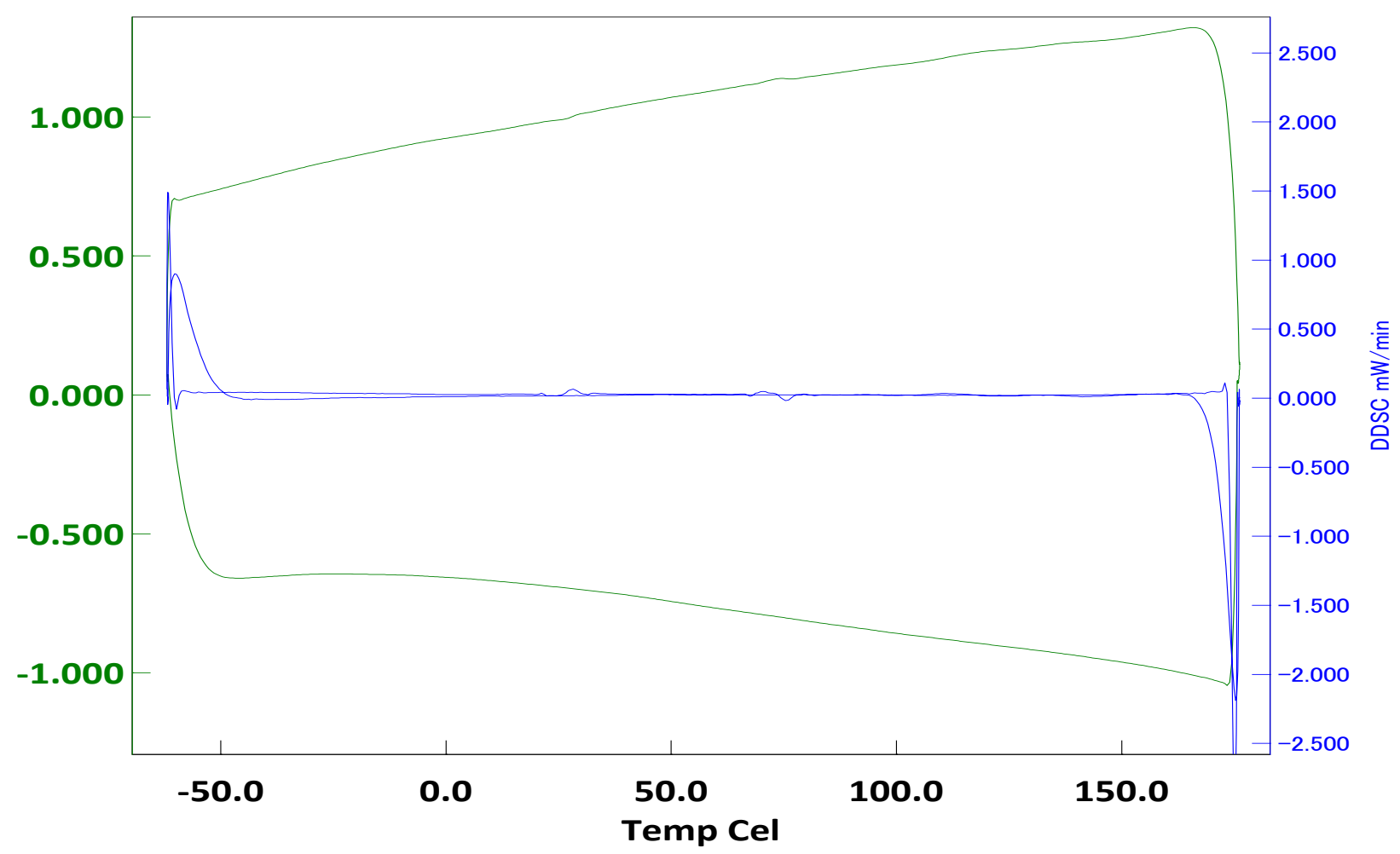

Figure S33. DSC chart of aminolysis product $\mathbf{3} \mathbf{b}_{\mathbf{1}}$ 


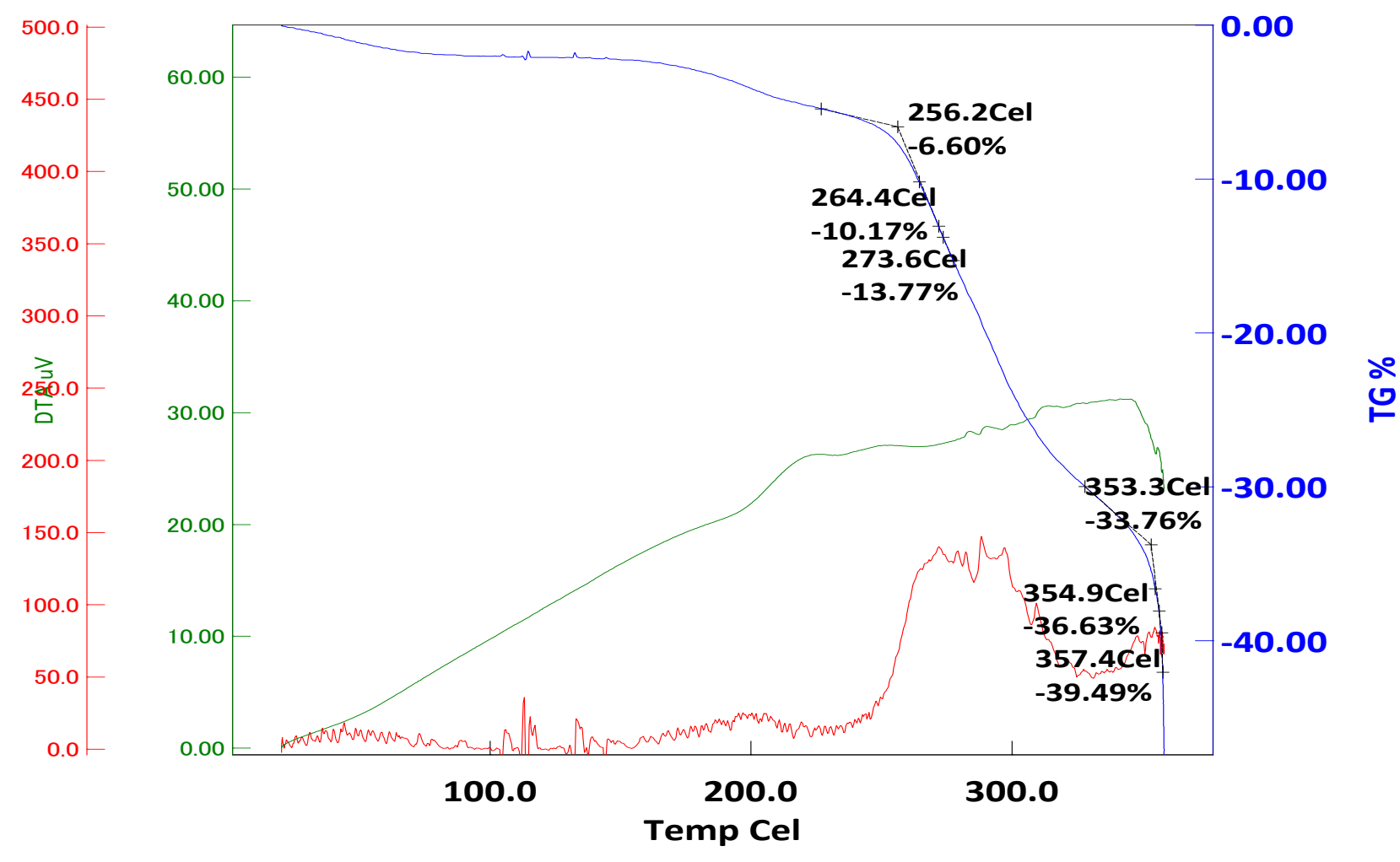

Figure S34. TG chart of aminolysis product $\mathbf{3} \mathbf{b}_{2}$

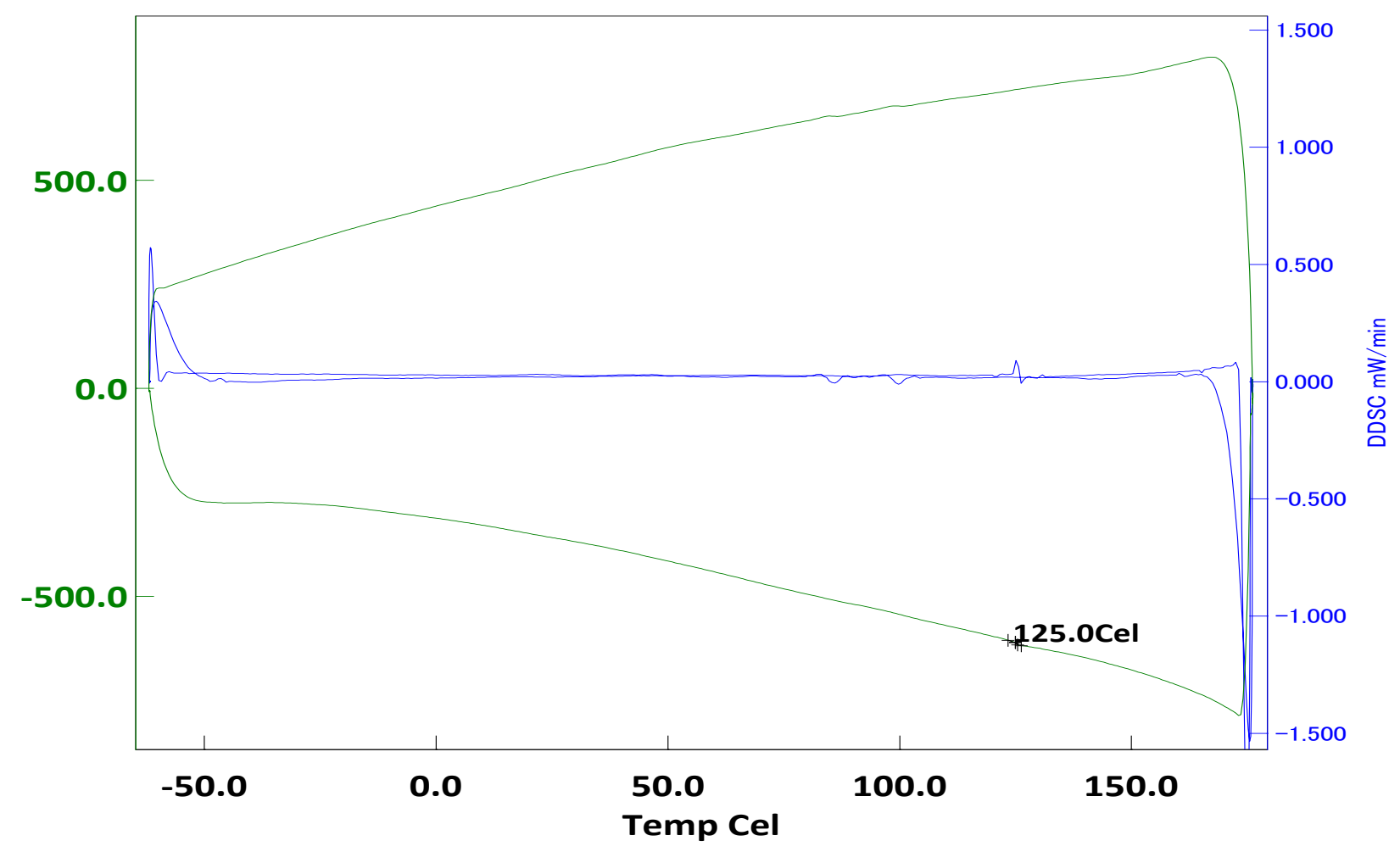

Figure S35. DSC chart of aminolysis product $\mathbf{3} \mathbf{b}_{2}$ 


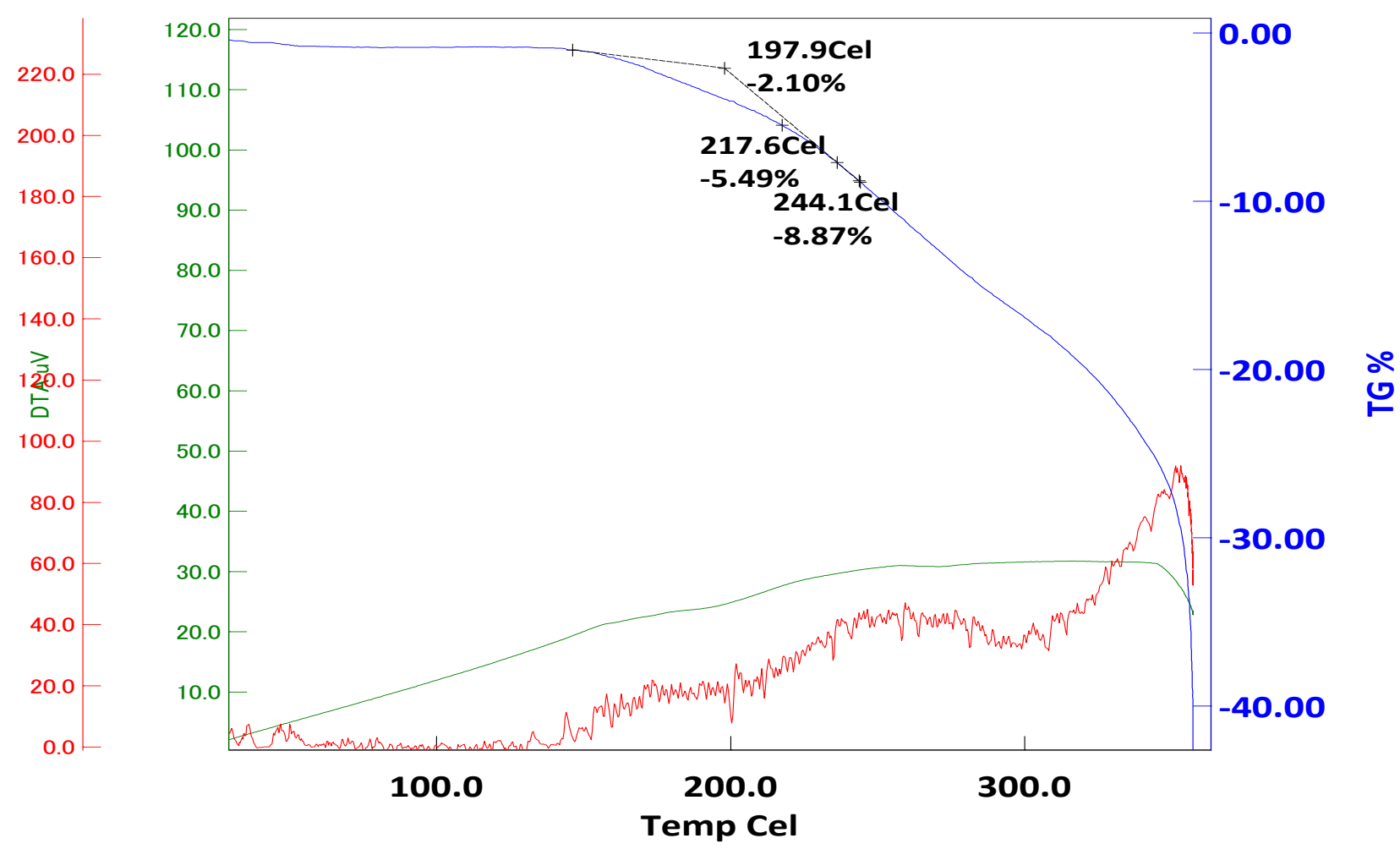

Figure S36. TG chart of ring-closed aminolysis product $\mathbf{5} \mathbf{b}_{\mathbf{1}}$

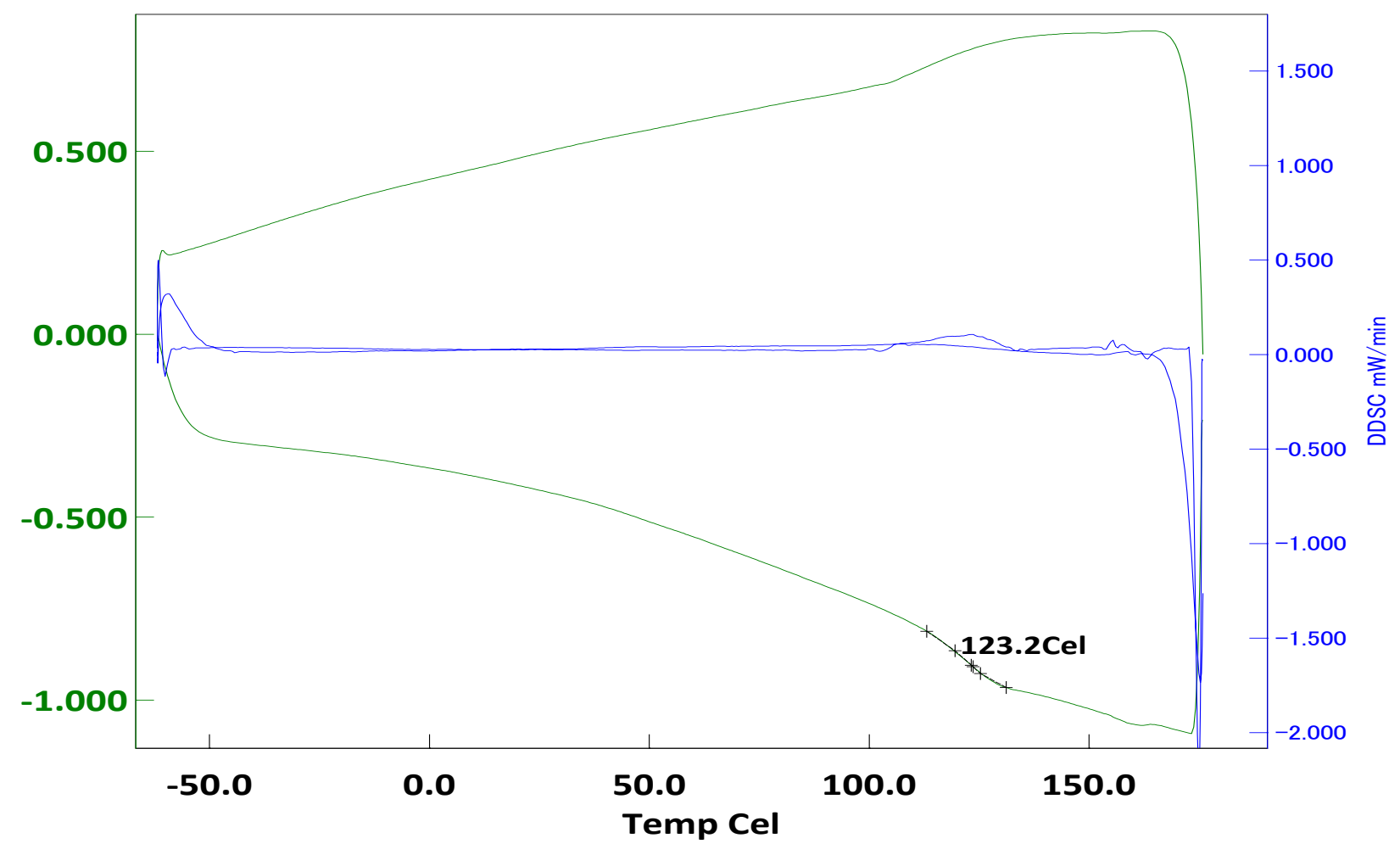

Figure S34. DSC chart of ring-closed aminolysis product $\mathbf{5} \mathbf{b}_{\mathbf{1}}$ 